Petersson, A., Kemp, A.I.S., and Whitehouse, M.J., 2019, A Yilgarn seed to the Pilbara Craton (Australia)? Evidence from inherited zircons: Geology, v. 47, https://doi.org/10.1130/G46696.1

\title{
SAMPLE LOCATIONS
}

\begin{tabular}{|l|c|r|r|}
\hline Sample name & UTM Zone & UTM Easting & UTM Northing \\
\hline 15 TKPB17 & $50 \mathrm{~K}$ & 748275 & 7646526 \\
\hline 18 APPB12 & $50 \mathrm{~K}$ & 747991 & 7646382 \\
\hline 18 APPB13 & $50 \mathrm{~K}$ & 748044 & 7646393 \\
\hline 18 APPB16 & $50 \mathrm{~K}$ & 750833 & 7646782 \\
\hline
\end{tabular}

\section{ANALYTICAL METHODS}

\section{Major And Trace-Element Analyses}

Two of the samples (15TKPB17 and 18APPB16) were analysed by Bureau Veritas Minerals Pty Ltd in Perth. The samples were cast using a 66:34 (Lithium Tetraborate 66\% / Lithium Metaborate 34\%) flux with 4\% Lithium nitrate added to form a glass bead. Major elements were determined by X-Ray Fluorescence. Trace elements were determined by Laser Ablation Inductively Coupled Plasma Mass Spectrometry on a fused bead. Loss on Ignition was determined using a robotic TGA system with furnaces set to $110^{\circ} \mathrm{C}$ and $1000^{\circ} \mathrm{C}$ (LOI1000). A sub-sample was digested with sulphuric and hydrofluoric acids and $\mathrm{FeO}$ determined via titration. Accuracy and precision of the analyses were monitored using geochemical reference rock powders (Kerba Monzogranite and Bunbury Basalt; Morris, 2007), inserted as unknown samples. All major and trace element data, including that obtained from the reference materials, are found in Supplementary table DR2.

\section{Zircon U-Pb SIMS Age Determination Nordsim}

Secondary ion mass spectrometry (SIMS) U-Th-Pb isotopic analyses were performed using a large geometry Cameca IMS1280 mass spectrometer at the Swedish Museum of Natural History. The instrument set up follows that of Whitehouse et al. (1999), Whitehouse and Kamber (2005) and references therein. An $\mathrm{O}_{2}$-primary beam with $23 \mathrm{kV}$ incident energy $(-13$ $\mathrm{kV}$ primary, $+10 \mathrm{kV}$ secondary) was used for sputtering. For this study, the primary beam was operated in aperture illumination (Köhler) mode yielding a 15-20 $\mu \mathrm{m}$ spot. Presputtering with a $25 \mu \mathrm{m}$ raster for $120 \mathrm{~s}$, centring of the secondary ion beam in the $3000 \mu \mathrm{m}$ field aperture (FA), mass calibration optimisation, and optimisation of the secondary beam energy distribution was performed automatically for each run, FA, and energy adjustment using the ${ }^{90} \mathrm{Zr}_{2}{ }^{16} \mathrm{O}^{+}$species at nominal mass 196 . Mass calibration of all peaks in the monocollection sequence was performed at the start of each session; within run mass calibration optimisation was performed using the ${ }^{90} \mathrm{Zr}_{2}{ }^{16} \mathrm{O}^{+}$peak, applying a shift to all other species. A mass resolution $(\mathrm{M} / \Delta \mathrm{M})$ of $\sim 5400$ was used to ensure adequate separation of $\mathrm{Pb}$ isotope peaks from nearby $\mathrm{HfSi}^{+}$species. Ion signals were detected using the axial ion-counting electron multiplier. All analyses were run in fully automated chain sequences. Data reduction 
assumes a power law relationship between $\mathrm{Pb}^{+} / \mathrm{U}^{+}$and $\mathrm{UO}_{2}{ }^{+} / \mathrm{U}^{+}$ratios with an empirically derived slope in order to calculate actual $\mathrm{Pb} / \mathrm{U}$ ratios based on those in the 91500 standard. $\mathrm{U}$ concentration and $\mathrm{Th} / \mathrm{U}$ ratio are also referenced to the 91500 standard. Common $\mathrm{Pb}$ correction is made if the ${ }^{204} \mathrm{~Pb}$ signal statistically exceeds average background and assumes a ${ }^{207} \mathrm{~Pb} /{ }^{206} \mathrm{~Pb}$ ratio of 0.83 (equivalent to present day Stacey and Kramers, 1975, model terrestrial $\mathrm{Pb}$ ). Decay constants follow the recommendations of Jaffey et al., (1971) and compiled by Steiger and Jäger (1977). All age calculations were done in Isoplot 4.15 (Ludwig, 2008) and quoted at $2 \sigma$ uncertainty level. All internal errors and external uncertainty in the standard analyses are propagated. Systematic uncertainties highlighted by interlaboratory experiments are not propagated. All results are presented in Supplementary table DR1.

\section{Curtin SHRIMP}

Zircon ion microprobe dating on the SHRIMP II at Curtin University, Perth, follow the methodology of De Laeter and Kennedy (1998), Kennedy and De Laeter (1998), and Nelson et al. (1997). All zircon U-Pb isotope data is presented in Supplementary table DR1. All ${ }^{207} \mathrm{~Pb} /{ }^{206} \mathrm{~Pb}$ zircon ages referred to in this study have been corrected for common $\mathrm{Pb}$, assuming a composition of common $\mathrm{Pb}$ following Compston et al. (1984). Reference zircon M257 $\left({ }^{206} \mathrm{~Pb} /{ }^{238} \mathrm{U}\right.$ age $=561.3 \pm 0.6 \mathrm{Ma}$; Nasdala et al., 2008) was used as primary standard and OGC zircons $\left({ }^{207} \mathrm{~Pb} /{ }^{206} \mathrm{~Pb}\right.$ age $=3465.4 \pm 0.6 \mathrm{Ma}$, Stern et al., 2009) were routinely analyzed to monitor analytical precision and accuracy of ${ }^{207} \mathrm{~Pb} /{ }^{206} \mathrm{~Pb}$. All SHRIMP U-Pb data were reduced via the software packages Isoplot and Squid (Ludwig, 2008; 2009).

\section{Ion Microprobe Oxygen Isotope Analysis In Zircon}

Oxygen isotope ratios $\left({ }^{18} \mathrm{O} /{ }^{16} \mathrm{O}\right)$ in zircon were determined using a Cameca IMS 1280 multicollector ion microprobe hosted by the Centre for Microscopy, Characterisation and Analysis (CMCA), University of Western Australia (UWA). After U-Pb analyses, Au-coating was removed using a light polishing. The sample mounts were then carefully cleaned with detergent, distilled water and ethanol in an ultrasonic bath and re-coated with gold $(30 \mathrm{~nm}$ in thickness) prior to SIMS O isotope analyses.

The sample surface is sputtered over a $10 \times 10 \mu \mathrm{m}$ area with a $10 \mathrm{kV}$, Gaussian $\mathrm{Cs}^{+}$beam with an intensity of $\sim 3 \mathrm{nA}$ and total impact energy of $20 \mathrm{keV}$. An electron gun is used to ensure charge compensation during the analyses. Secondary ions were admitted in the double focusing mass spectrometer within a $110 \mu \mathrm{m}$ entrance slit and focused in the centre of a 4000 $\mu \mathrm{m}$ field aperture (x 100 magnification). They are energy filtered using a $30 \mathrm{eV}$ band pass with a $5 \mathrm{eV}$ gap toward the high-energy side. ${ }^{16} \mathrm{O}$ and ${ }^{18} \mathrm{O}$ are collected simultaneously in Faraday cup detectors fitted with $10^{10} \Omega$ (L'2) and $10^{11} \Omega(\mathrm{H} 1)$ resistors, respectively, and operating at a mass resolution of $\sim 2430$. The magnetic field was regulated using NMR control.

Each analysis includes a pre-sputtering over a $15 \times 15 \mu \mathrm{m}$ area during $30 \mathrm{~s}$ and the automatic centring of the secondary ions in the field aperture, contrast aperture and entrance slit. Each analysis then consists of 20 four-second cycles, which give an average internal precision of $\sim 0.16 \%$ o ( $2 \mathrm{SE}$ ). The analytical session was monitored in terms of drift using at least two bracketing standards every 5 to 6 sample analyses. Instrumental mass fractionation (IMF) was corrected using Temora 2 (8.2\%; Black et al., 2004) following the procedures described in Kita et al. (2009). The spot-to-spot reproducibility was $0.2-0.3 \%$ (2 SD) on Temora 2 during the analytical session. Two extra zircon references were used for quality control, Penglai $\left(\mathrm{n}=26\right.$, weighted mean ${ }^{18} \mathrm{O} /{ }^{16} \mathrm{O}=0.0020154 \pm 0.0000001$, reference ${ }^{18} \mathrm{O} /{ }^{16} \mathrm{O}=0.0020158 \pm 0.0000002$ (Li et al., 2010) and $\mathrm{OGC} / \mathrm{OG} 1(\mathrm{n}=32$, weighted mean 
${ }^{18} \mathrm{O} /{ }^{16} \mathrm{O}=0.0020171 \pm 0.0000001$, reference ${ }^{18} \mathrm{O} /{ }^{16} \mathrm{O}=0.0020170 \pm 0.0000001$ (Petersson et al., 2019). Uncertainty on each $\delta^{18} \mathrm{O}$ spot has been calculated by propagating the errors on instrumental mass fractionation determination, which include the standard deviation of the mean oxygen isotope ratio measured on the primary standard during the session, and internal error on each sample data point. Raw ${ }^{18} \mathrm{O} /{ }^{16} \mathrm{O}$ ratios and corrected $\delta^{18} \mathrm{O}$ (quoted with respect to Vienna Standard Mean Ocean Water or VSMOW) are found in Supplementary table DR3 and DR4.

\section{LA-MC-ICP-MS Zircon Lu-Hf Analyses}

$\mathrm{Lu}-\mathrm{Hf}$ analyses were carried out at the School of Earth Sciences at The University of Western Australia using a $193 \mathrm{~nm}$ Cetac Analyte G2 excimer laser installed with a twovolume HelEx2 sample cell, and a Thermo-Scientific Neptune Plus High-Resolution Multicollector ICP-MS.

$\mathrm{Lu}-\mathrm{Hf}$ spot overlapped pits from both the $\mathrm{U}-\mathrm{Pb}$ and $\mathrm{O}$ isotope analysis wherever possible. Circular spots with a diameter of 40-50 $\mu \mathrm{m}$ were used. Each analysis was initiated by a $30 \mathrm{~s}$ electronic baseline followed by an ablation sequence of $60 \mathrm{~s}$ comprising 60 integration cycles of one second each. A laser pulse repetition rate of $4 \mathrm{~Hz}$ was used and the laser energy was held at $\sim 5 \mathrm{~J} / \mathrm{cm}^{2}$, which equals an ablation rate of $\sim 0.05 \mu \mathrm{m}$ per pulse for zircon. Helium carrier gas $(1.0 \mathrm{l} / \mathrm{min})$ was used to transport the ablated particles from the sample chamber. This was combined with argon gas (flow rate c. $0.61 / \mathrm{min})$ and nitrogen $(\sim 0.0121 / \mathrm{min})$ further downstream before entering the argon plasma. Masses ${ }^{171} \mathrm{Yb},{ }^{173} \mathrm{Yb},{ }^{175} \mathrm{Lu},{ }^{176}(\mathrm{Hf}+\mathrm{Lu}+\mathrm{Yb})$, ${ }^{177} \mathrm{Hf},{ }^{178} \mathrm{Hf},{ }^{179} \mathrm{Hf}$ and ${ }^{180}(\mathrm{Hf}+\mathrm{W}+\mathrm{Ta})$ were measured simultaneously by Faraday detectors. Isobaric interference of ${ }^{176} \mathrm{Yb}$ and ${ }^{176} \mathrm{Lu}$ on ${ }^{176} \mathrm{Hf}$ was calculated using the measured intensities of ${ }^{171} \mathrm{Yb}$ and ${ }^{175} \mathrm{Lu}$ along with known isotopic ratios of ${ }^{176} \mathrm{Yb} /{ }^{171} \mathrm{Yb}=0.897145$ (Segal et al., 2003) and ${ }^{176} \mathrm{Lu} /{ }^{175} \mathrm{Lu}=0.02655$ (Vervoort et al., 2004). Mass bias corrections were calculated using the exponential law. For calculations of $\beta \mathrm{Hf}$, measured intensities of ${ }^{179} \mathrm{Hf}$ and ${ }^{177} \mathrm{Hf}$ and a ${ }^{179} \mathrm{Hf} /{ }^{177} \mathrm{Hf}$ ratio of 0.7325 was used. $\beta \mathrm{Yb}$ was calculated using measured intensities of ${ }^{173} \mathrm{Yb}$ and ${ }^{171} \mathrm{Yb}$ and a ${ }^{176} \mathrm{Yb} /{ }^{171} \mathrm{Yb}$ ratio of 1.130172 (Segal et al., 2003). Mass bias behaviour of Lu was assumed to be identical to $\mathrm{Yb}$. Four zircon references were used for quality control, FC- $1\left({ }^{176} \mathrm{Hf} /{ }^{177} \mathrm{Hf}=0.282179 \pm 0.000019\right.$ at $2 \mathrm{SD}, \mathrm{n}=16$, weighted average ${ }^{176} \mathrm{Hf} /{ }^{177} \mathrm{Hf}=0.282179 \pm 0.000005$ at $95 \%$ conf. MSWD $=0.9$; solution value $0.282184 \pm 0.000016)$, Mud Tank zircon $\left({ }^{176} \mathrm{Hf} /{ }^{177} \mathrm{Hf}=0.282491 \pm 0.000011, \mathrm{n}=26\right.$, weighted average ${ }^{176} \mathrm{Hf} /{ }^{177} \mathrm{Hf}=0.282490 \pm 0.000003$ at $95 \%$ conf. $\mathrm{MSWD}=1.8$; solution value $0.282507 \pm 0.000006$, Temora $2\left({ }^{176} \mathrm{Hf} /{ }^{177} \mathrm{Hf}=0.282683 \pm 0.000020, \mathrm{n}=3\right.$; weighted average ${ }^{176} \mathrm{Hf} /{ }^{177} \mathrm{Hf}=0.282683 \pm 0.000011$ at $95 \%$ conf. $\mathrm{MSWD}=0.3$; solution value $0.282686 \pm 0.000008), 91500\left({ }^{176} \mathrm{Hf} /{ }^{177} \mathrm{Hf}=0.282305 \pm 0.000023, \mathrm{n}=8\right.$; weighted average ${ }^{176} \mathrm{Hf} /{ }^{177} \mathrm{Hf}=0.282306 \pm 0.000008$ at $95 \%$ conf. MSWD $=0.6$; solution value $0.282306 \pm 0.000008$, Woodhead and Hergt, 2005) and OGC

$\left({ }^{176} \mathrm{Hf} /{ }^{177} \mathrm{Hf}=0.280635 \pm 0.000020, \mathrm{n}=15\right.$; weighted average ${ }^{176} \mathrm{Hf} /{ }^{177} \mathrm{Hf}=0.280633 \pm 0.000014$ at $95 \%$ conf. $\mathrm{MSWD}=6.2$; solution value $0.280633 \pm 0.000034$, Kemp et al., 2017). Agecorrected ${ }^{176} \mathrm{Hf} /{ }^{177} \mathrm{Hf}_{(3.467 \mathrm{Ga})}$ for the high ${ }^{176} \mathrm{Lu} /{ }^{177} \mathrm{Hf}$ zircon yields a weighted mean of ${ }^{176} \mathrm{Hf} /{ }^{177} \mathrm{Hf}=0.280557 \pm 0.000005$, at $95 \%$ conf., $\mathrm{MSWD}=0.6$, within errors identical to the solution value ${ }^{176} \mathrm{Hf} /{ }^{177} \mathrm{Hf}=0.280554 \pm 0.000007$ of Kemp et al., (2017). Analysed ${ }^{176} \mathrm{Hf} /{ }^{177} \mathrm{Hf}$ ratios of the sample zircon were normalised based on a comparison between the mean of analysed ${ }^{176} \mathrm{Hf} /{ }^{177} \mathrm{Hf}$ ratios of Mud Tank zircon measured in a given session and its reported ${ }^{176} \mathrm{Hf} /{ }^{177} \mathrm{Hf}$ ratio of 0.282507 determined by solution analysis (Woodhead and Hergt, 2005) and itself reported relative to $\mathrm{JMC} 475{ }^{176} \mathrm{Hf} /{ }^{177} \mathrm{Hf}=0.282160$ ). Calculations of $\varepsilon \mathrm{Hf}$ were done using $\lambda^{176} \mathrm{Lu}=1.867 \times 10^{-11} \mathrm{yr}^{-1}$ (Scherer et al., 2001; Söderlund et al., 2004), $\left({ }^{176} \mathrm{Lu} /{ }^{177} \mathrm{Hf}\right) \mathrm{CHUR}=0.0336$ and $\left({ }^{176} \mathrm{Hf} /{ }^{177} \mathrm{Hf}\right) \mathrm{CHUR}=0.282785 \pm 11$

(Bouvier et al., 2008). All zircon Lu-Hf data is found in Supplementary table DR5 and DR6. 


\section{References}

Black, L.P., Kamo, S.L., Allen, C.M., Davis, D.W., Aleinikoff, J.N., Valley, J.W., Mundil, R., Campbell, I.H., Korsch, R.J.,Williams, I.S., and Foudoulis, C., 2004, Improved ${ }^{206} \mathrm{~Pb} /{ }^{238} \mathrm{U}$ microprobe geochronology by the monitoring of a trace-element-related matrix effect; SHRIMP, ID-TIMS, ELA-ICP-MS and oxygen isotope documentation for a series of zircon standards: Chemical Geology, v. 205, p. 115-140.

https://doi.org/10.1016/j.chemgeo.2004.01.003

Bouvier, A., Vervoort, J.D., and Patchett, P.J., 2008, The Lu-Hf and Sm-Nd isotopic composition of CHUR: constraints from unequilibrated chondrites and implication for the bulk composition of terrestrial planets: Earth and Planetary Science Letters, v. 273, p. 48-57. https://doi.org/10.1016/j.epsl.2008.06.010

Compston, W., Williams, I.S., and Meyer, C., 1984, U-Pb geochronology of zircons from lunar breccia 73217 using a sensitive high mass-resolution ion microprobe: Journal of Geophysical Research: Solid Earth, v. 89, p. B525-B534.

https://doi.org/10.1029/JB089iS02p0B525

De Laeter, J.R., and Kennedy A.K., 1998, A double focusing mass spectrometer for geochronology: International Journal of Mass Spectrometry, v. 78, p. 43-50. https://doi.org/10.1016/S1387-3806(98)14092-7

Jaffey, A.H., Flynn, K.F., Glendenin, L.E., Bentley, W.T., and Essling, A.M., 1971, Precision measurement of half-lives and specific activities of $U 235$ and $U$ 238: Physical Review $C$, 4(5), p.1889. https://doi.org/10.1103/PhysRevC.4.1889

Kemp, A.I., Vervoort, J.D., Bjorkman, K.E., and Iaccheri, L.M., 2017, Hafnium Isotope Characteristics of Palaeoarchaean Zircon OG1/OGC from the Owens Gully Diorite, Pilbara Craton, Western Australia: Geostandards and Geoanalytical Research, v. 41, 659-673. https://doi.org/10.1111/ggr.12182

Kennedy, A.K., and de Laeter, J.R., 1994, The performance characteristics of the WA SHRIMP II ion microprobe. In: Eight International Conference on Geochronology, Cosmochronology and Isotope Geology. Berkley, USA. Abstracts Vol., U.S. Geological Survey Circular, v. 1107, p. 166.

Kita, N.T., Ushikubo, T., Fu, B., and Valley, J.W., 2009, High precision SIMS oxygen isotope analysis and the effect of sample topography: Chemical Geology, v. 264, p. 43-57. https://doi.org/10.1016/j.chemgeo.2009.02.012

Li, X.H., Long, W.G., Li, Q.L., Liu, Y., Zheng, Y.F., Yang, Y.H., Chamberlain, K.R., Wan, D.F., Guo, C.H., Wang, X.C., and Tao, H., 2010, Penglai zircon megacrysts: a potential new working reference material for microbeam determination of $\mathrm{Hf}-\mathrm{O}$ isotopes and $\mathrm{U}-\mathrm{Pb}$ age: Geostandards and Geoanalytical Research, v. 34(2), p.117-134.

https://doi.org/10.1111/j.1751-908X.2010.00036.X

Ludwig, K.R., 2008, Isoplot 3.70. A Geochronological Toolkit for Microsoft Excel:

Berkeley Geochronology Center Special Publications, pp. 4. 
Ludwig, K.R., 2009, SQUID II., a user's manual, Berkeley Geochronology Center: Special Publication 2, 2455 Ridge Road, Berkeley, CA 94709, USA 22.

Morris, PA, 2007, Composition of the Bunbury basalt (BB1) and Kerba Monzogranite (KG1) geochemical reference materials, and assessing the contamination effects of mill heads: Geological Survey of Western Australia, Record 2007/14, 22p.

Nasdala, L., Hofmeister, W., Norberg, N., Martinson, J.M., Corfu, F., Dörr, W., Kamo, S.L., Kennedy, A.K., Kronz, A., Reiners, P.W., Frei, D., Kosler, J., Wan, Y., Götze, J., Häger, T., Kröner, A., and Valley, J.W., 2008, Zircon M257 - a Homogeneous Natural Reference Material for the Ion Microprobe U-Pb Analysis of Zircon: Geostandards and Geoanalytical Research, v. 32, p. 247-265. https://doi.org/10.1111/j.1751-908X.2008.00914.X

Nelson, D.R., 1997, Compilation of SHRIMP U-Pb zircon geochronology data, 1996. Geological Survey of Western Australia Record No. 1997/2.

Petersson, A., Kemp, A.I., Hickman, A.H., Whitehouse, M.J., Martin, L., and Gray, C.M., 2019, A new 3.59 Ga magmatic suite and a chondritic source to the east Pilbara Craton: Chemical Geology, v. 511, p. 51-70. https://doi.org/10.1016/j.chemgeo.2019.01.021

Scherer, E., Münker, C., and Mezger, K., 2001, Calibration of the lutetium-hafnium clock: Science, v. 293, p. 683-687. http://doi.org/10.1126/science.1061372

Segal, I., Halicz, L., and Platzner, I.T., 2003, Accurate isotope ratio measurements of ytterbium by multiple collector inductively coupled plasma mass spectrometry applying erbium and hafnium in an improved double external normalization procedure: Journal of Analytical Atomic Spectrometry, v. 18, p. 1217-1223. https://doi.org/10.1039/B307016F

Stacey, J.S., and Kramers, J.D., 1975, Approximation of terrestrial lead isotope evolution by a 2-stage model: Earth and Planetary Science Letters, v, 26, p. 207-221.

https://doi.org/10.1016/0012-821X(75)90088-6

Steiger, R.H., and Jäger, E., 1977, Subcommission on geochronology: convention of the use of decay constants in geo- and cosmochronology: Earth and Planetary Science Letters, v. 36, p. 359-362. https://doi.org/10.1016/0012-821X(77)90060-7

Stern, R.A., Bodorkos, S., Kamo, S.L., Hickman, A.H., and Corfu, F., 2009, Measurement of SIMS Instrumental Mass Fractionation of Pb Isotopes During Zircon Dating: Geostandards and Geoanalytical Research, v. 33, p. 145-168, http://doi.org/10.1111/j.1751908X.2009.00023.X.

Söderlund, U., Patchett, P.J., Vervoort, J.D., and Isachsen, C.E., 2004, The 176Lu decay constant determined by Lu-Hf and U-Pb isotope systematics of Precambrian mafic instrusions: Earth and Planetary Science Letters, v. 219, p. 311-324.

https://doi.org/10.1016/S0012-821X(04)00012-3

Vervoort, J.D., Patchett, P.J., Söderlund, U., and Baker, M., 2004, Isotopic composition of $\mathrm{Yb}$ and the determination of $\mathrm{Lu}$ concentrations and $\mathrm{Lu} / \mathrm{Hf}$ ratios by isotope dilution using MC-ICPMS. Geochemistry, Geophysics, Geosystems, v. 5(11). https://doi.org/10.1029/2004GC000721 
Whitehouse, M.J., Kamber, B.S., and Moorbath, S., 1999, Age significance of U-Th-Pb zircon data from early Archean rocks of west Greenland - a reassessment based on combined ion microprobe and imaging studies: Chemical Geology, v. 160, p. 210-224.

https://doi.org/10.1016/S0009-2541(99)00066-2

Whitehouse, M.J., and Kamber, B.S., 2005, Assigning dates to thin gneissic veins in highgrade metamorphic terranes: a cautionary tale from Akilia, Southwest Greenland: Journal of Petrology, v. 46, p. 291-318. https://doi.org/10.1093/petrology/egh075

Woodhead, J.D., and Hergt, J.M., 2005, A preliminary appraisal of seven natural zircon reference materials for in situ Hf isotope determination: Geostandards and Geoanalytical Research, v. 29, p. 183-195. https://doi.org/10.1111/j.1751-908X.2005.tb00891.X 

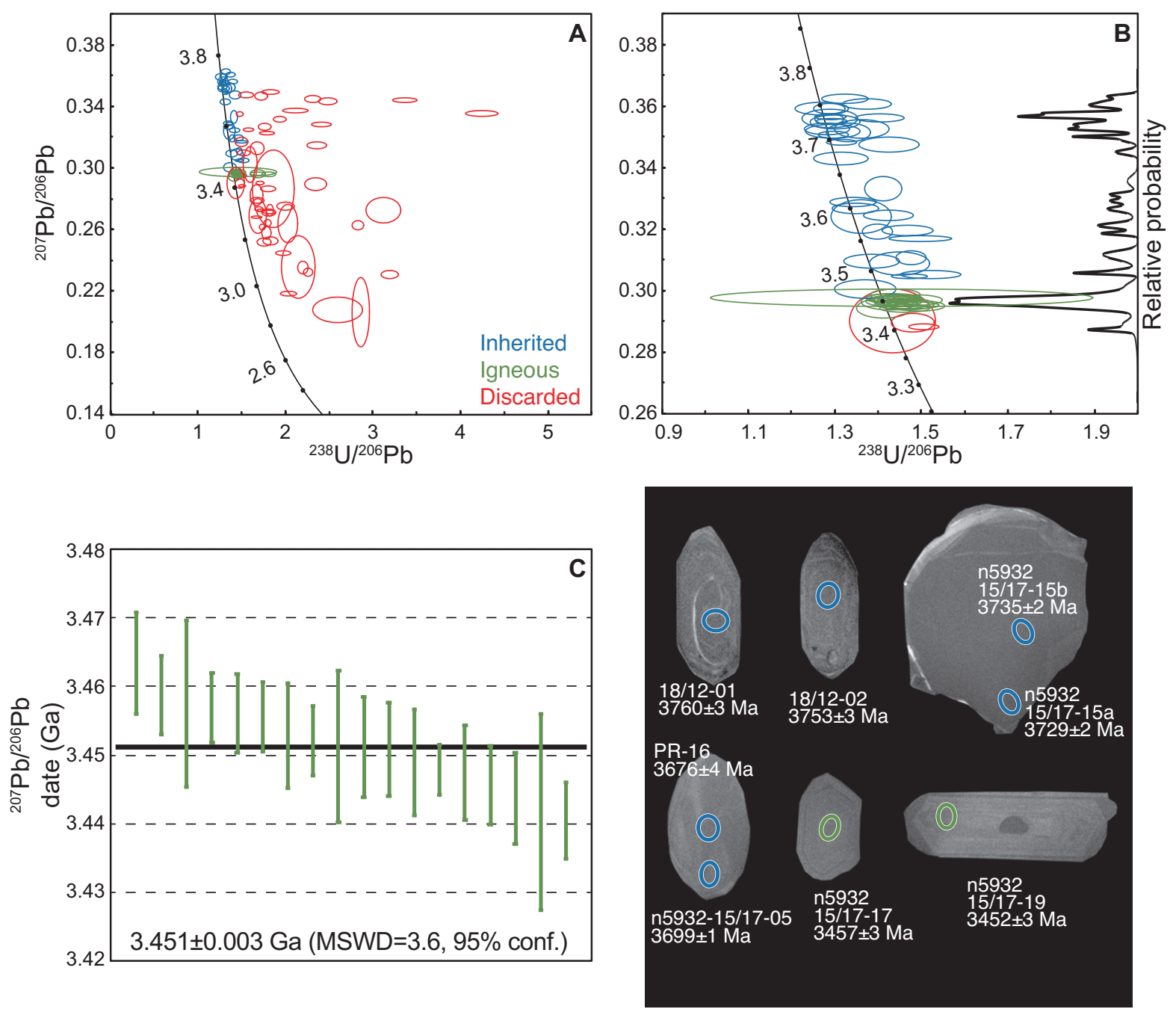

Fig. DR1 A: Tera-Wasserburg diagrams showing SIMS (Secondary-Ion-MassSpectrometry) zircon spot data for all zircon U-Pb analyses. B: Ellipses denote all $<10 \%$ discordant zircon $\mathrm{U}-\mathrm{Pb}$ analyses. $\mathrm{C}:{ }^{207} \mathrm{~Pb} /{ }^{206} \mathrm{~Pb}$ weighted mean of igneous grains. Lower right panel: Cathodoluminescence $(C L)$ images of representative zircon grains. Ellipses indicate SIMS spot locations for U-Pb. Blue denote inherited, green denote igneous and red denote discarded analysis. 


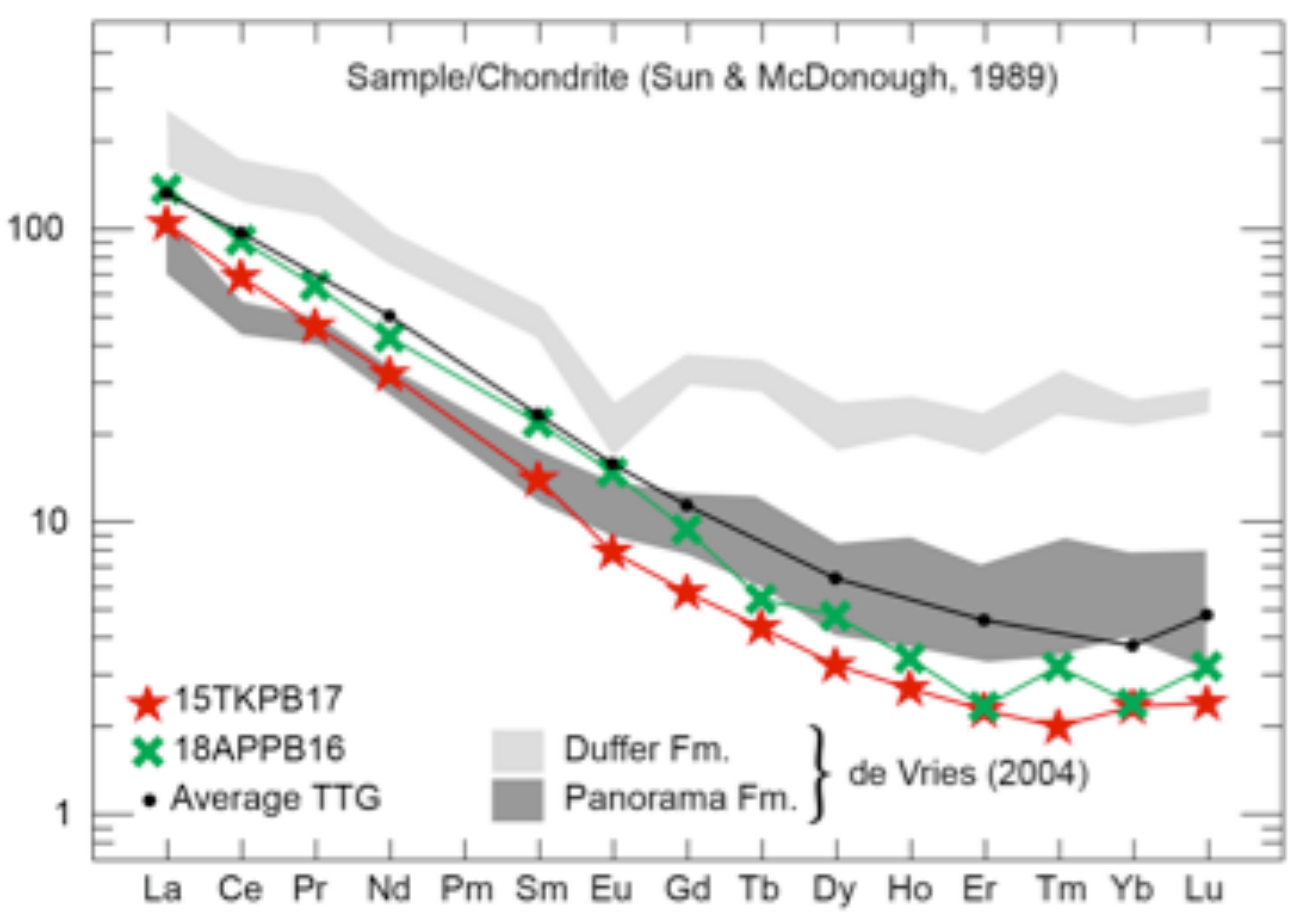

Fig. DR2 The majority of Panorama Ridge is actually mapped as Duffer Formation on the latest map published by the Geological survey of Western Australia (Hickman, 2012). There seem to be a statistically reliable difference in age between the two units where the Duffer Formation is dated to 3.474-3.463 Ga (McNaughton et al. 1993; Nelson 2001) and the ca. 3.458-3.428 Ga (Thorpe et al. 1992; Nelson 1998) age of the Panorama Formation, here constrained to 3.451 \pm 3 Ma. Hickman

(1983) argued that, similarly to pre- and post-tectonic granitic rocks, the Duffer and Whyman Formations differ in that the Duffer formation is dacitic while the Whyman formation is rhyolitic. Similarly, De Vries (2004) pointed out a few key differences in REE patterns between the Duffer and Panorama formations, where the Duffer dacites have Eu-anomalies and virtually flat HREE (Gd-Lu), while the Panorama Formation rhyodacites have lower REE abundances and lack an Eu-anomaly. The REE signature of the samples in this study confirm a Panorama Formation affiliation (DR2). Further, the new and improved age presented here reinforces a Panorama formation association to the sampled unit.

De Vries, S.T., 2004. Early Archaean sedimentary basins: depositional environment and hydrothermal systems (Doctoral dissertation, University Utrecht). http://dspace.library.uu.nl/handle/1874/1155

Hickman, A.H., 1983. Geology of the Pilbara Block and its environs. West. Aust. Geol. Surv. Bull. 127, $268 \mathrm{p}$.

McNaughton, N.J., Compston, W. and Barley, M.E., 1993. Constraints on the age of the Warrawoona Group, eastern Pilbara block, Western Australia. Precambrian Research, 60(1-4), pp.69-98. https://doi.org/10.1016/0301-9268(93)90045-4

Nelson, D.R., 2001, Compilation of geochronology data, 2000: Geological Survey of Western Australia Record 2001/2, 205 p.

Nelson, D.R., 1998, Compilation of SHRIMP U-Pb zircon geochronology data, 1997: Geological Survey of Western Australia Record 1998/2, 242 p.

Thorpe, R.I., Hickman, A.H., Davis, D.W., Mortensen, J.K. and Trendall, A.F., 1992. U-Pb zircon geochronology of Archaean felsic units in the Marble Bar region, Pilbara Craton, Western Australia. Precambrian Research, 56(3-4), pp.169-189. https://doi.org/10.1016/0301-9268(92)90100-3 


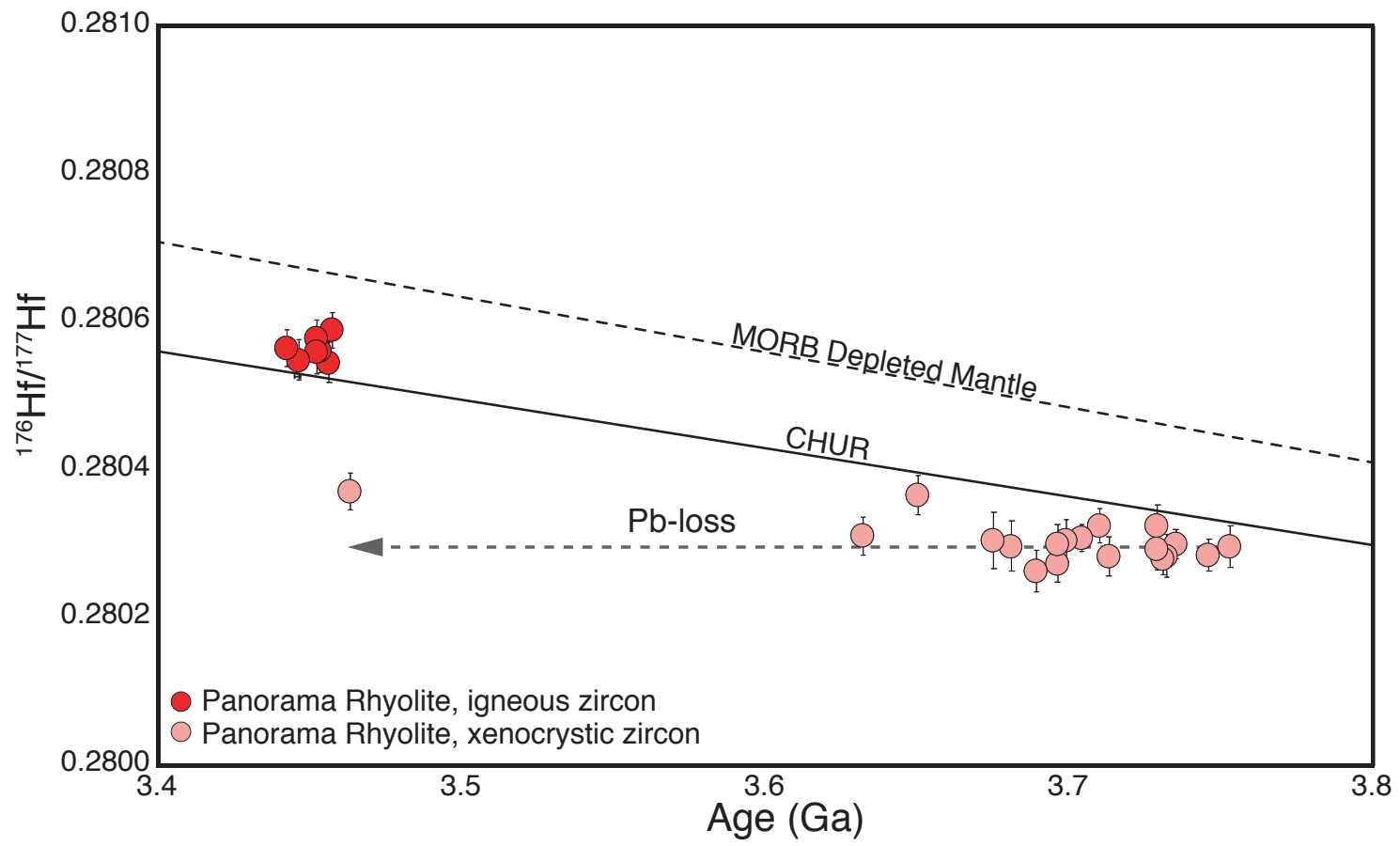

Fig. DR3 


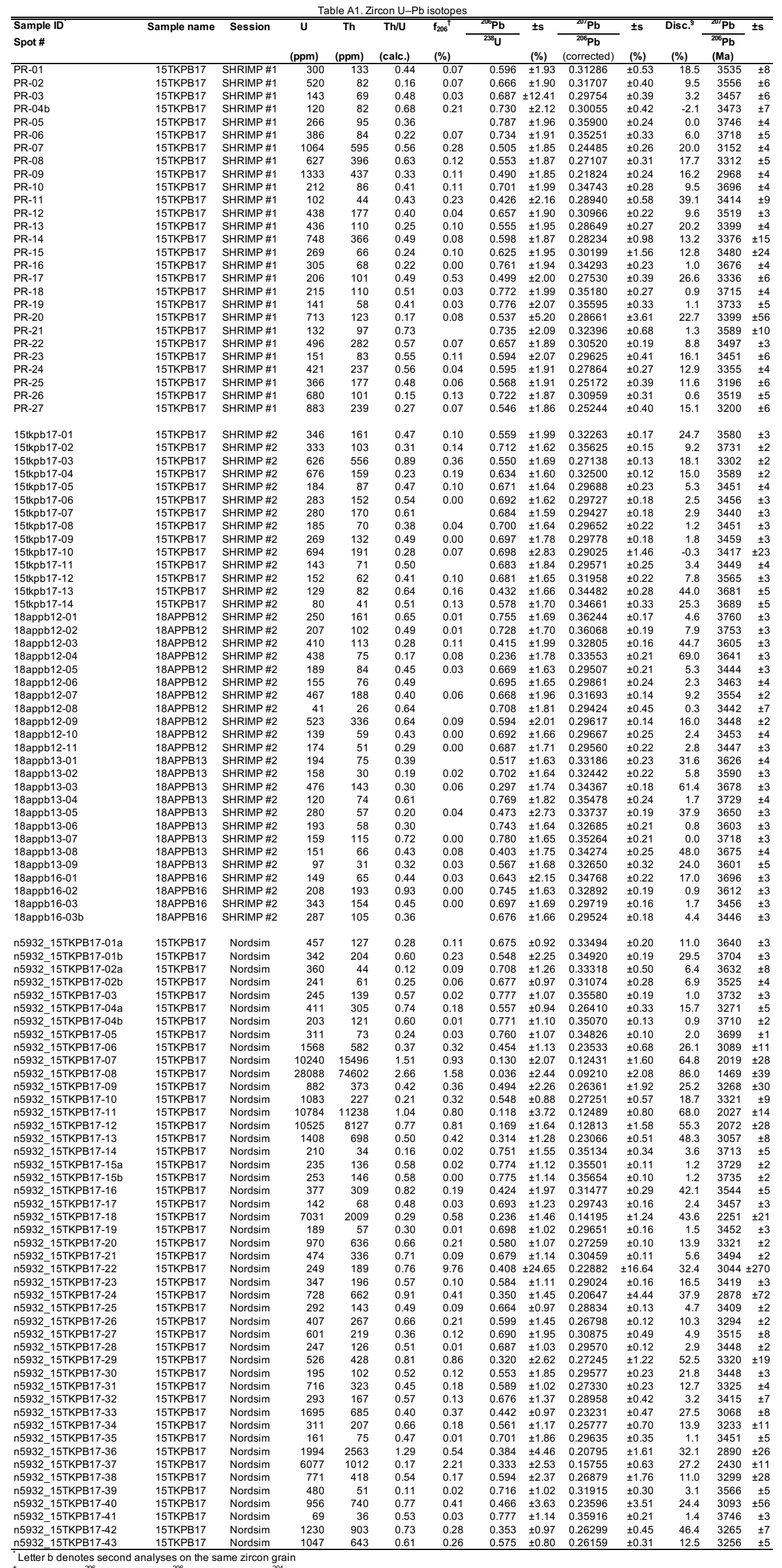

\% of common ${ }^{206} \mathrm{~Pb}$ in measured ${ }^{206} \mathrm{~Pb}$, estimated from ${ }^{204} \mathrm{~Pb}$ assuming a present day Stacey and Kramers (1975) model for terrestrial Pb-isotope composition.

${ }^{\S}$ Age discordance in conventional concordia space. Negative numbers are reverse discordant. 
Table A2. Major and Trace element data

\begin{tabular}{|c|c|c|c|c|c|c|}
\hline Sample & 15TKPB17 & 18APPB16 & Kerba monzogranite & $\mathrm{KG}^{*}{ }^{*}$ & "Bunbury Basalt (BB1) & $\mathrm{BB}^{*}{ }^{*}$ \\
\hline \multicolumn{7}{|c|}{ Major elements } \\
\hline $\mathrm{SiO}_{2}$ & 82.1 & 84.3 & 71.50 & 71.37 & 52.20 & 51.82 \\
\hline $\mathrm{TiO}_{2}$ & 0.14 & 0.17 & 0.24 & 0.24 & 2.04 & 2.02 \\
\hline $\mathrm{Al}_{2} \mathrm{O}_{3}$ & 10.4 & 12.6 & 15.00 & 14.95 & 15.40 & 15.33 \\
\hline $\mathrm{Fe}_{2} \mathrm{O}_{3}$ & 0.2 & 0.1 & 0.72 & 0.65 & 3.55 & 3.28 \\
\hline $\mathrm{FeO}$ & 0.0 & 0.0 & 1.19 & 1.29 & 7.78 & 7.99 \\
\hline $\mathrm{FeO}^{\text {tot }}$ & 0.24 & 0.07 & & & & \\
\hline $\mathrm{MgO}$ & 0.74 & 0.02 & 0.04 & 0.04 & 0.16 & 0.16 \\
\hline $\mathrm{MnO}$ & $\mathrm{n} / \mathrm{a}$ & $\mathrm{n} / \mathrm{a}$ & 0.48 & 0.48 & 4.63 & 4.72 \\
\hline $\mathrm{CaO}$ & 0.69 & 0.00 & 2.14 & 2.16 & 8.66 & 8.71 \\
\hline $\mathrm{Na}_{2} \mathrm{O}$ & 0.07 & 0.09 & 4.2 & 4.07 & 3.09 & 3.03 \\
\hline $\mathrm{K}_{2} \mathrm{O}$ & 2.99 & 0.293 & 3.48 & 3.47 & 0.45 & 0.46 \\
\hline $\mathrm{P}_{2} \mathrm{O}_{5}$ & 0.013 & 0.025 & 0.064 & 0.08 & 0.242 & 0.25 \\
\hline $\mathrm{SO}_{3}$ & 0.02 & $\mathrm{n} / \mathrm{a}$ & 0.015 & 0.03 & 0.154 & 0.16 \\
\hline $\mathrm{BaO}$ & 0.113 & 0.0475 & 0.166 & 0.0137 & 0.017 & 0.015 \\
\hline $\mathrm{LOI}_{\text {tot }}$ & 2.6 & 2.32 & 0.5 & & 0.34 & \\
\hline Total & 100.1 & 99.9 & 99.73 & & 98.71 & \\
\hline \multicolumn{7}{|c|}{ Trace elements } \\
\hline $\mathrm{Ba}$ & 113 & 47.5 & 1420 & 1373 & 168 & 152 \\
\hline $\mathrm{Rb}$ & 62.2 & 7.1 & 90.5 & 96 & 12.1 & 12.4 \\
\hline $\mathrm{Sr}$ & 24.7 & 31.5 & 564 & 515 & 273 & 245 \\
\hline $\mathrm{Zr}$ & 105 & 122 & 175 & 190 & 154 & 153 \\
\hline $\mathrm{Nb}$ & 2.9 & 3.38 & 8.68 & 9.5 & 6.87 & 7.6 \\
\hline Y & 3.82 & 4.88 & 4.66 & 4.5 & 41.9 & 39.8 \\
\hline Sc & 3.1 & $\mathrm{n} / \mathrm{a}$ & 1.4 & 2.4 & 26.7 & 27.7 \\
\hline V & 12.5 & 9.6 & 14 & 14.6 & 248 & 245 \\
\hline $\mathrm{Cr}$ & 2 & $\mathrm{n} / \mathrm{a}$ & 161 & 170 & 146 & 150 \\
\hline Co & 19.9 & 23.4 & 2.8 & - & 36.3 & 37 \\
\hline $\mathrm{Ni}$ & 4 & 4 & 6 & 8 & 54 & 41 \\
\hline $\mathrm{Cu}$ & $\mathrm{n} / \mathrm{a}$ & $\mathrm{n} / \mathrm{a}$ & 4 & 6.2 & 80 & 82 \\
\hline $\mathrm{Zn}$ & $\mathrm{n} / \mathrm{a}$ & $\mathrm{n} / \mathrm{a}$ & 50 & 41 & 130 & 116 \\
\hline $\mathrm{Ga}$ & 15.9 & 17.8 & 18.4 & 20 & 23.5 & 23 \\
\hline La & 24.4 & 31.9 & 43.9 & 43.8 & 11.6 & 10.95 \\
\hline $\mathrm{Ce}$ & 41.3 & 55.1 & 69.2 & 71.3 & 27.9 & 26.8 \\
\hline $\operatorname{Pr}$ & 4.36 & 5.98 & 7.1 & 6.6 & 4.1 & 3.6 \\
\hline $\mathrm{Nd}$ & 14.7 & 19.6 & 21.9 & 22.4 & 19.9 & 18.75 \\
\hline $\mathrm{Sm}$ & 2.1 & 3.28 & 3.25 & 2.8 & 6.06 & 5.69 \\
\hline $\mathrm{Eu}$ & 0.45 & 0.84 & 0.73 & 0.8 & 1.94 & 2.01 \\
\hline Gd & 1.16 & 1.9 & 1.63 & 1.5 & 7.29 & 7.26 \\
\hline $\mathrm{Tb}$ & 0.16 & 0.2 & 0.18 & 0.2 & 1.21 & 1.22 \\
\hline Dy & 0.81 & 1.19 & 0.78 & 0.8 & 7.27 & 7.27 \\
\hline $\mathrm{Ho}$ & 0.15 & 0.19 & 0.15 & 0.1 & 1.51 & 1.46 \\
\hline $\mathrm{Er}$ & 0.37 & 0.38 & 0.4 & 0.4 & 4.04 & 3.95 \\
\hline Tm & 0.05 & 0.08 & 0.07 & - & 0.56 & 0.53 \\
\hline $\mathrm{Yb}$ & 0.39 & 0.4 & 0.44 & 0.5 & 3.36 & 3.32 \\
\hline Lu & 0.06 & 0.08 & 0.08 & 0.1 & 0.48 & 0.49 \\
\hline $\mathrm{Hf}$ & 2.83 & 3.44 & 4.62 & 5 & 4.04 & 4 \\
\hline Тa & 0.4 & 0.52 & 0.93 & 1 & 0.44 & 0.5 \\
\hline $\mathrm{Pb}$ & 5 & 3 & 38 & 40 & 4 & 4 \\
\hline Th & 7.84 & 10.6 & 15.8 & 17.4 & 1.63 & 1.8 \\
\hline U & 2.04 & 1.78 & 3.74 & 4.2 & 0.29 & 0.3 \\
\hline
\end{tabular}

Note: Majors have been determined by X-Ray Fluorescence Spectrometry.

Traces have been determined by Laser Ablation Inductively Coupled Plasma Mass Spectrometry.

Loss on Ignition results have been determined using a robotic TGA system at $1000^{\circ} \mathrm{C}$.

Ferrous Iron $(\mathrm{FeO})$ content was determined by acid digestion followed by volumetric titration.

$\mathrm{FeO}^{\text {tot }}=\mathrm{FeO}+0.8998^{*} \mathrm{Fe}_{2} \mathrm{O}_{3}$

*Suggested reference-values for the Kerba monzogranite and the Bunbury Basalt from, Morris (2007). 
Table A3. Zircon O-isotopes

\begin{tabular}{|c|c|c|c|c|c|c|c|}
\hline Spot I.D & $\begin{array}{c}\text { Corresponding } \\
\text { U-Pb spot I.D }\end{array}$ & Comment & $\begin{array}{l}\text { Sequenti } \\
\text { al } \\
\text { no." }\end{array}$ & $\begin{array}{l}\text { Drift, back-ground } \\
\text { and SIMS } \\
\text { corrected }\end{array}$ & abs. & $\delta^{18} O^{5}$ & abs. \\
\hline & & & & ${ }^{18} \mathrm{O}^{16} \mathrm{O}^{\dagger}$ & $1 \mathrm{~s}$ & $(\%)$ & $2 \mathrm{~s}$ \\
\hline Cameca IMS 128 & multi-collector ion & croprobe Centr & e for Micros & scopy, Characterisa & tion and Analy & sis, Un & iversity \\
\hline Session I & & & & & & & \\
\hline$\frac{15 \text { TKPB } 17}{15 \text { TKPB17-01 }}$ & $\frac{\text { Corresp. U-Pb }}{15 \text { tkpb17-03 }}$ & Small crack & 7 & 0.0020128 & \pm 0.00000046 & 3.78 & \pm 0.45 \\
\hline 15TKPB 17-02 & 15tkpb17-09 & ok & 8 & 0.0020167 & \pm 0.00000047 & 5.71 & \pm 0.47 \\
\hline 15TKPB17-03 & 15tkpb17-08 & ok & 9 & 0.0020160 & \pm 0.00000048 & 5.39 & \pm 0.48 \\
\hline 15TKPB17-04 & 15tkpb $17-02$ & ok & 10 & 0.0020151 & \pm 0.00000050 & 4.94 & \pm 0.50 \\
\hline 15TKPB17-04b & 15tkpb17-02 & ok & 11 & 0.0020164 & \pm 0.00000046 & 5.59 & \pm 0.46 \\
\hline 15TKPB $17-05$ & 15tkpb17-07 & ok & 16 & 0.0020166 & \pm 0.00000050 & 5.69 & \pm 0.50 \\
\hline 15TKPB17-06 & 15tkpb17-01 & Crack & 17 & 0.0020151 & \pm 0.00000050 & 4.92 & \pm 0.50 \\
\hline $15 T K P B 17-06 b$ & 15tkpb17-01 & Crack & 18 & 0.0020140 & \pm 0.00000045 & 4.40 & \pm 0.45 \\
\hline 15TKPB17-06c & 15tkpb17-01 & ok & 19 & 0.0020160 & \pm 0.00000046 & 5.37 & \pm 0.46 \\
\hline 15TKPB17-07 & & ok & 20 & 0.0020160 & \pm 0.00000057 & 5.40 & \pm 0.57 \\
\hline 15TKPB17-08 & 15tkpb17-05 & ok & 23 & 0.0020168 & \pm 0.00000040 & 5.79 & \pm 0.40 \\
\hline 15TKPB17-09 & 15tkpb17-04 & Metamict zone & 24 & 0.0020130 & \pm 0.00000046 & 3.88 & \pm 0.46 \\
\hline 15TKPB17-09b & 15tkpb17-04 & Metamict zone & 25 & 0.0020140 & \pm 0.00000046 & 4.38 & \pm 0.46 \\
\hline 15TKPB17-10 & & ok & 26 & 0.0020173 & \pm 0.00000043 & 6.05 & \pm 0.43 \\
\hline 15TKPB17-11 & & ok & 27 & 0.0020179 & \pm 0.00000046 & 6.33 & \pm 0.46 \\
\hline 15TKPB17-12 & & ok & 28 & 0.0020158 & \pm 0.00000053 & 5.30 & \pm 0.53 \\
\hline 15TKPB17-13 & & ok & 33 & 0.0020171 & \pm 0.00000045 & 5.93 & \pm 0.45 \\
\hline 15TKPB17-14 & & ok & 34 & 0.0020163 & \pm 0.00000043 & 5.55 & \pm 0.43 \\
\hline 15TKPB 17-15 & & ok & 35 & 0.0020167 & \pm 0.00000053 & 5.73 & \pm 0.53 \\
\hline 15TKPB17-15b & & ok & 36 & 0.0020175 & \pm 0.00000050 & 6.13 & \pm 0.50 \\
\hline 15TKPB $17-16$ & & ok & 37 & 0.0020171 & \pm 0.00000049 & 5.92 & \pm 0.49 \\
\hline Weighted averag & & & & & & 5.70 & \pm 0.25 \\
\hline 18APPB 12 & & & & & & & \\
\hline 18APPB12-01 & 18appb12-01 & ok & 117 & 0.0020160 & \pm 0.00000053 & 5.36 & \pm 0.53 \\
\hline 18APPB12-01b & 18appb12-01 & ok & 118 & 0.0020161 & \pm 0.00000047 & 5.42 & \pm 0.47 \\
\hline 18APPB $12-02$ & 18appb12-02 & Crack & 121 & 0.0020144 & \pm 0.00000058 & 4.57 & \pm 0.57 \\
\hline 18APPB $12-02 b$ & 18appb12-02 & Crack & 122 & 0.0020124 & \pm 0.00000042 & 3.57 & \pm 0.42 \\
\hline $18 A P P B 12-03$ & 18appb12-10 & Crack & 123 & 0.0020174 & \pm 0.00000055 & 6.08 & \pm 0.55 \\
\hline 18APPB $12-04$ & & ok & 124 & 0.0020168 & \pm 0.00000048 & 5.79 & \pm 0.48 \\
\hline 18APPB12-05 & 18appb12-08 & ok & 125 & 0.0020174 & \pm 0.00000043 & 6.10 & \pm 0.43 \\
\hline 18APPB12-05b & 18appb12-08 & ok & 126 & 0.0020182 & \pm 0.00000042 & 6.47 & \pm 0.42 \\
\hline 18APPB 12-06 & 18appb12-06 & ok & 127 & 0.0020184 & \pm 0.00000047 & 6.57 & \pm 0.47 \\
\hline 18APPB12-07 & 18appb12-03 & ok & 131 & 0.0020178 & \pm 0.00000059 & 6.27 & \pm 0.59 \\
\hline 18APPB 12-08 & & ok & 132 & 0.0020173 & \pm 0.00000056 & 6.05 & \pm 0.56 \\
\hline 18APPB12-09 & 18appb12-04 & ok & 133 & 0.0020159 & \pm 0.00000052 & 5.31 & \pm 0.52 \\
\hline 18APPB $12-10$ & 18appb12-05 & ok & 134 & 0.0020172 & \pm 0.00000051 & 5.97 & \pm 0.50 \\
\hline 18APPB12-10b & 18appb12-05 & ok & 135 & 0.0020168 & \pm 0.00000051 & 5.80 & \pm 0.51 \\
\hline 18APPB 12-11 & 18appb12-11 & ok & 136 & 0.0020172 & \pm 0.00000051 & 6.00 & \pm 0.51 \\
\hline Weighted averag & & & & & & 5.95 & \pm 0.31 \\
\hline 18APPB 13 & & & & & & & \\
\hline 18APPB13-01 & & ok & 41 & 0.0020190 & \pm 0.00000047 & 6.87 & \pm 0.47 \\
\hline 18APPB 13-02 & & ok & 42 & 0.0020173 & \pm 0.00000054 & 6.03 & \pm 0.54 \\
\hline 18APPB 13-03 & 18appb13-06 & ok & 43 & 0.0020164 & \pm 0.00000047 & 5.57 & \pm 0.46 \\
\hline 18APPB13-03b & 18appb13-06 & ok & 44 & 0.0020158 & \pm 0.00000047 & 5.29 & \pm 0.47 \\
\hline 18APPB 13-04 & 18appb13-07 & ok & 45 & 0.0020157 & \pm 0.00000048 & 5.24 & \pm 0.48 \\
\hline 18APPB13-04b & 18appb13-07 & ok & 46 & 0.0020148 & \pm 0.00000048 & 4.78 & \pm 0.48 \\
\hline 18APPB 13-05 & & ok & 51 & 0.0020177 & \pm 0.00000050 & 6.24 & $\begin{array}{l} \pm 0.49 \\
\pm 0.49\end{array}$ \\
\hline 18APPB 13-06 & & ok & 52 & 0.0020161 & \pm 0.00000047 & 5.42 & \pm 0.47 \\
\hline 18APPB13-07 & 18appb13-08 & Big crack & 53 & 0.0020213 & \pm 0.00000044 & 8.02 & \pm 0.44 \\
\hline 18APPB13-07b & 18appb13-08 & ok & 54 & 0.0020134 & \pm 0.00000051 & 4.11 & \pm 0.50 \\
\hline 18APPB 13-08 & 18appb13-09 & ok & 55 & 0.0020166 & \pm 0.00000045 & 5.69 & \pm 0.45 \\
\hline 18APPB 13-09 & 18appb13-05 & ok & 59 & 0.0020163 & \pm 0.00000049 & 5.52 & \pm 0.49 \\
\hline 18APPB $13-10$ & 18appb13-04 & Crack & 60 & 0.0020149 & \pm 0.00000040 & 4.84 & \pm 0.40 \\
\hline 18APPB13-10b & 18appb13-04 & ok & 61 & 0.0020160 & \pm 0.00000045 & 5.36 & \pm 0.45 \\
\hline 18APPB $13-11$ & & ok & 62 & 0.0020164 & \pm 0.00000048 & 5.57 & \pm 0.48 \\
\hline 18APPB13-12 & & Crack & 63 & 0.0020253 & \pm 0.00000047 & 10.01 & \pm 0.47 \\
\hline 18APPB $13-13$ & & ok & 64 & 0.0020173 & \pm 0.00000049 & 6.02 & \pm 0.49 \\
\hline 18APPB13-14 & 18appb13-03 & ok & 68 & 0.0020180 & \pm 0.00000049 & 6.39 & \pm 0.48 \\
\hline 18APPB13-15 & & ok & 69 & 0.0020183 & \pm 0.00000052 & 6.51 & \pm 0.52 \\
\hline 18APPB13-15b & & ok & 70 & 0.0020179 & \pm 0.00000051 & 6.35 & \pm 0.51 \\
\hline 18APPB 13-16 & & Crack & 71 & 0.0020195 & \pm 0.00000054 & 7.15 & \pm 0.54 \\
\hline 18APPB 13-17 & & ok & 72 & 0.0020172 & \pm 0.00000050 & 5.99 & \pm 0.50 \\
\hline 18APPB13-04 & 18appb13-02 & ok & 73 & 0.0020156 & \pm 0.00000045 & 5.20 & \pm 0.45 \\
\hline 18APPB 13-05 & & ok & 77 & 0.0020156 & \pm 0.00000048 & 5.18 & \pm 0.47 \\
\hline 18APPB 13-04 & & ok & 78 & 0.0020171 & \pm 0.00000056 & 5.94 & \pm 0.56 \\
\hline Weighted averag & & & & & & 5.73 & \pm 0.23 \\
\hline 18APPB 16 & & & & & & & \\
\hline 18APPB16-01 & 18appb16-01 & ok & 88 & 0.0020166 & \pm 0.00000045 & 5.68 & \pm 0.45 \\
\hline 18APPB 16-01b & 18appb16-01 & Big crack & 89 & 0.0020222 & \pm 0.00000050 & 8.48 & \pm 0.50 \\
\hline 18APPB $16-02$ & & ok & 90 & 0.0020172 & \pm 0.00000045 & 5.97 & \pm 0.45 \\
\hline 18APPB 16-03 & & ok & 91 & 0.0020179 & \pm 0.00000050 & 6.32 & \pm 0.50 \\
\hline 18APPB $16-05$ & & Big crack & 96 & 0.0020202 & \pm 0.00000048 & 7.46 & \pm 0.48 \\
\hline 18APPB16-05b & & Crack & 97 & 0.0020177 & \pm 0.00000053 & 6.23 & \pm 0.53 \\
\hline 18APPB $16-06$ & 18appb16-02 & ok & 98 & 0.0020177 & \pm 0.00000043 & 6.22 & \pm 0.43 \\
\hline 18APPB $16-07$ & & ok & 99 & 0.0020163 & \pm 0.00000043 & 5.56 & \pm 0.43 \\
\hline 18APPB 16-08 & 18appb16-03 & ok & 100 & 0.0020180 & $\begin{array}{l} \pm .00000049 \\
\end{array}$ & 6.37 & \pm 0.49 \\
\hline 18APPB 16-08b & 18appb16-03 & ok & 104 & 0.0020175 & \pm 0.00000047 & 6.11 & \pm 0.47 \\
\hline 18APPB $16-09$ & & ok & 105 & 0.0020168 & \pm 0.00000050 & 5.79 & \pm 0.50 \\
\hline 18APPB16-10 & & ok & 106 & 0.0020169 & \pm 0.00000052 & 5.84 & \pm 0.52 \\
\hline 18APPB $16-10 b$ & & ok & 107 & 0.0020169 & \pm 0.00000049 & 5.85 & \pm 0.49 \\
\hline 18APPB $16-11$ & & ok & 108 & 0.0020176 & \pm 0.00000046 & 6.18 & \pm 0.46 \\
\hline 18APPB $16-12$ & & ok & 109 & 0.0020170 & \pm 0.00000051 & 5.86 & \pm 0.51 \\
\hline 18APPB16-12b & & ok & 113 & 0.0020169 & \pm 0.00000049 & 5.82 & \pm 0.49 \\
\hline 18APPB $16-13$ & & ok & 114 & 0.0020173 & \pm 0.00000053 & 6.02 & \pm 0.53 \\
\hline 18APPB16-13b & & ok & 115 & 0.0020173 & \pm 0.00000054 & 6.03 & \pm 0.54 \\
\hline 18APPB $16-14$ & & ok & 116 & 0.0020168 & \pm 0.00000050 & 5.77 & \pm 0.50 \\
\hline Weighted avera & & & & & & 5.96 & \pm 0.26 \\
\hline
\end{tabular}


Session II

15TKPB17

15TKPB17

15TKPB17-02

15TKPB17-03

5TKPB17-04

15TKPB17-05

5TKPB17-06

15TKPB17-07

15TKPB17-08

15TKPB17-09
15 TKPB17-09b

15TKPB17-09b

15TKPB17-10

15TKPB17-11

15TKPB17-11b
15TKPB17-11c

15TKPB17-11c
15TKPB 17-11d

15TKPB17-12

n5932-15TKPB17-35 Crack

15TKPB17-13

15TKPB $17-13$

Weighted average

n5932_15TKPB17-17 Crack

ก5932_15TKPB17-01t o

n5932_15TKPB17-30 ok

5932_15TKPB17-28 ok

25932 15TKPB17-03 ok

5932_15TKPB17-19 ok

PR-18 15 ok

5932_15TKPB17-41 Crack

55932_ 15TKPB17-02t ok

ก5932_15TKPB17-15; ok

5932_15TKPB17-15t ok

15TKPB17-15k

Weighted average n5932_15TKPB17 ok 15 TKPB17 Session

15TKPB17 Session I \& II combined

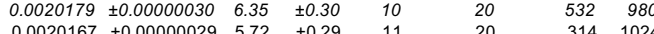

$\begin{array}{lllll}314 & 1024 & 3457 \pm 3 & 0.48 & 2.4\end{array}$

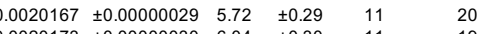

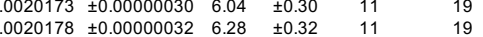

$\begin{array}{rlllllllllrr}0.0020176 & \pm 0.00000032 & 6.17 & \pm 0.32 & 11 & 20 & -480 & 2457 & 3448 \pm 2 & 0.51 & 2.9\end{array}$

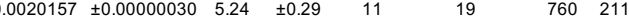

$3732 \pm 3 \quad 0.57 \quad 1.0$

$766 \quad 2165$

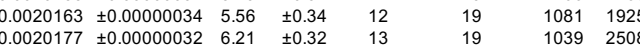

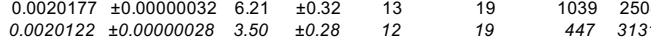

$\begin{array}{llllllll}0.0020122 & \pm 0.00000028 & 3.50 & \pm 0.28 & 12 & 19 & 447 & 3131 \\ 0.0020138 & +0.00000030 & 4.26 & \pm 0.30 & 12 & 19 & 426 & 3154\end{array}$

$\begin{array}{rrrrrrrr}0.0020138 & \pm 0.00000030 & 4.26 & \pm 0.30 & 12 & 19 & 426 & 3154 \\ 0.0020173 & \pm 0.00000031 & 6.04 & \pm 0.31 & 12 & 17 & 1224 & 1588\end{array}$

$\begin{array}{lll}3732 \pm 3 & 0.57 & 1.0\end{array}$

$\begin{array}{lll}3452 \pm 3 & 0.31 & 1.5 \\ 3715 \pm 4 & 0.51 & 0.9\end{array}$

$\begin{array}{lll}3746 \pm 3 & 0.53 & 1.4\end{array}$

$\begin{array}{lll}3746 \pm 3 & 0.53 \quad 1.4\end{array}$

$\begin{array}{lllll}1361 & 1226 & 3729 \pm 2 & 0.59 & 1.2 \\ 1387 & 122 & 3735 \pm 2 & 0.58\end{array}$

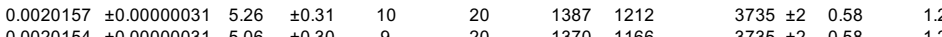

$\begin{array}{lllllllllll}0.0020154 & \pm 0.00000031 & 5.06 & \pm 0.30 & 9 & 20 & 1370 & 1166 & 3735 \pm 2 & 0.58 & 1.2\end{array}$

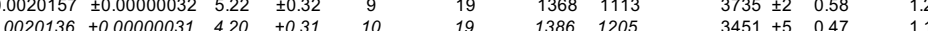

$\begin{array}{lllllllllll}0.0020174 & \pm 0.00000030 & 6.07 & \pm 0.29 & 8 & 20 & 1641 & 501 & 3451 \pm 5 & 0.47 & 1.1\end{array}$

$\begin{array}{llll}\text { All analyses both sessions } & 5.75 & \pm 0.10 \quad n=31 & M S D W=1.15\end{array}$

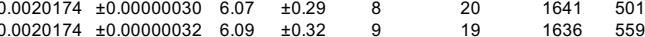

during the session,

Mount position $X Y$ for standard and sample indicates that most analyses are within the central region to standards.

Values in italic are interpreted to be unreliable due to sampling of discordant grains, inclusions, mixed domains, or on fractures.

Italic denotes discarded analyses.

If mutliple $\mathrm{U}-\mathrm{Pb}$ analyses on one grain, data from the nearest analyses is denoted in ${ }^{207} \mathrm{~Pb} / 207 \mathrm{~Pb}$, Th/U and $\mathrm{Discordance} \mathrm{columns.}$

Sequential order of analyses.

${ }^{\dagger}$ Raw ratios drift corrected using the standard analyses versus time. The instrumental mass fractionation, corrected using alpha SIMS (ratio between the session average standard value and reference $\delta_{\text {Normalised to a }} \delta^{18} \mathrm{O}$ value of $9.20 \%$ for the Temora 2 standard

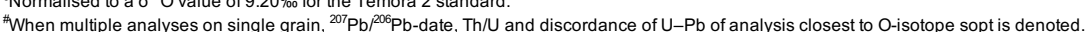

"Age discordance in conventional concordia space. Negative numbers are reverse discordant 
Table A4. Standrad zircon O-isotopes

\begin{tabular}{|c|c|c|c|c|c|c|c|c|c|}
\hline \multirow[t]{2}{*}{ Spot I.D } & \multirow[t]{2}{*}{$\begin{array}{l}\text { Sequential } \\
\text { no.* }\end{array}$} & \multirow{2}{*}{$\begin{array}{c}\text { Drift, back- } \\
\text { ground and } \\
\text { SIMS } \\
\text { corrected } \\
{ }^{18} \mathrm{O} /{ }^{16} \mathrm{O} \\
\end{array}$} & \multirow{2}{*}{$\begin{array}{c}\text { abs. } \\
1 \sigma \\
\end{array}$} & \multirow{2}{*}{$\begin{array}{c}\delta^{18} O^{\S} \\
(\% \circ) \\
\end{array}$} & \multirow{2}{*}{$\begin{array}{l}\text { abs. } \\
2 \sigma \\
\end{array}$} & \multicolumn{2}{|c|}{ Field aperture } & \multicolumn{2}{|c|}{ Mount location } \\
\hline & & & & & & $\mathbf{x}$ & $\mathbf{Y}$ & X-pos. & Y-pos. \\
\hline $\begin{array}{l}\text { Cameca IM } \\
\text { Session } \\
91500\end{array}$ & $\begin{array}{l}\text { IS } 1280 \mathrm{mu} \\
\text { I }\end{array}$ & -colle & croprobe C & ntre for $\mathrm{N}$ & $\mathrm{C}, \mathrm{Ch}$ & ation & & A, Perth. & \\
\hline $91500-01$ & 1 & 0.0020251 & \pm 0.0000005 & 9.93 & \pm 0.48 & -8 & 8 & 2912 & 836 \\
\hline $91500-02$ & 2 & 0.0020246 & \pm 0.0000005 & 9.66 & \pm 0.47 & -9 & 7 & 2952 & 836 \\
\hline $91500-03$ & 3 & 0.0020249 & \pm 0.0000005 & 9.80 & \pm 0.50 & -10 & 7 & 2898 & 796 \\
\hline $91500-04$ & 4 & 0.0020247 & \pm 0.0000005 & 9.74 & \pm 0.47 & -10 & 6 & 2938 & 796 \\
\hline $91500-05$ & 5 & 0.0020253 & \pm 0.0000005 & 10.01 & \pm 0.47 & -9 & 7 & 2938 & 756 \\
\hline $91500-07$ & 13 & 0.0020247 & \pm 0.0000005 & 9.73 & \pm 0.52 & -11 & 6 & 2892 & 610 \\
\hline 91500-09 & 21 & 0.0020252 & \pm 0.0000005 & 9.99 & \pm 0.49 & -11 & 5 & 2971 & 474 \\
\hline $91500-10$ & 29 & 0.0020249 & \pm 0.0000005 & 9.85 & \pm 0.53 & -11 & 5 & 3044 & 485 \\
\hline $91500-11$ & 30 & 0.0020249 & \pm 0.0000004 & 9.82 & \pm 0.44 & -11 & 5 & 3062 & 445 \\
\hline $91500-12$ & 38 & 0.0020243 & \pm 0.0000005 & 9.53 & \pm 0.47 & -11 & 4 & 3020 & 445 \\
\hline $91500-13$ & 39 & 0.0020247 & \pm 0.0000005 & 9.72 & \pm 0.46 & -11 & 4 & 2970 & 445 \\
\hline $91500-14$ & 47 & 0.0020243 & \pm 0.0000005 & 9.54 & \pm 0.45 & -11 & 5 & 2965 & 405 \\
\hline $91500-15$ & 48 & 0.0020254 & \pm 0.0000005 & 10.09 & \pm 0.46 & -11 & 5 & 3018 & 405 \\
\hline $91500-16$ & 56 & 0.0020242 & \pm 0.0000004 & 9.48 & \pm 0.45 & -11 & 5 & 3061 & 405 \\
\hline $91500-17$ & 57 & 0.0020251 & \pm 0.0000005 & 9.94 & \pm 0.45 & -11 & 5 & 3064 & 365 \\
\hline $91500-18$ & 65 & 0.0020247 & \pm 0.0000005 & 9.72 & \pm 0.50 & -11 & 4 & 3024 & 365 \\
\hline $91500-19$ & 66 & 0.0020248 & \pm 0.0000005 & 9.77 & \pm 0.48 & -11 & 4 & 2984 & 365 \\
\hline $91500-20$ & 74 & 0.0020256 & \pm 0.0000005 & 10.15 & \pm 0.46 & -12 & 3 & 2998 & 222 \\
\hline $91500-21$ & 75 & 0.0020253 & \pm 0.0000005 & 10.02 & \pm 0.52 & -11 & 3 & 3038 & 236 \\
\hline $91500-22$ & 83 & 0.0020254 & \pm 0.0000005 & 10.07 & \pm 0.53 & -12 & 3 & 3071 & 230 \\
\hline $91500-23$ & 84 & 0.0020246 & \pm 0.0000004 & 9.69 & \pm 0.44 & -12 & 4 & 3108 & 229 \\
\hline $91500-24$ & 92 & 0.0020253 & \pm 0.0000005 & 10.00 & \pm 0.46 & -11 & 3 & 3061 & 110 \\
\hline $91500-25$ & 93 & 0.0020253 & \pm 0.0000004 & 10.00 & \pm 0.44 & -11 & 3 & 3061 & 70 \\
\hline $91500-26$ & 101 & 0.0020253 & \pm 0.0000005 & 10.02 & \pm 0.54 & -10 & 3 & 3072 & 30 \\
\hline $91500-27$ & 102 & 0.0020256 & \pm 0.0000005 & 10.15 & \pm 0.46 & -10 & 4 & 3182 & 362 \\
\hline $91500-28$ & 110 & 0.0020250 & \pm 0.0000004 & 9.85 & \pm 0.44 & -10 & 4 & 3219 & 346 \\
\hline $91500-29$ & 111 & 0.0020254 & \pm 0.0000005 & 10.09 & \pm 0.46 & -9 & 4 & 3281 & 340 \\
\hline $91500-30$ & 119 & 0.0020252 & \pm 0.0000005 & 9.99 & \pm 0.47 & -10 & 3 & 3317 & 321 \\
\hline $91500-31$ & 120 & 0.0020249 & \pm 0.0000005 & 9.80 & \pm 0.52 & -9 & 5 & 3464 & 660 \\
\hline $91500-32$ & 128 & 0.0020245 & \pm 0.0000005 & 9.64 & \pm 0.53 & -9 & 5 & 3457 & 620 \\
\hline $91500-33$ & 129 & 0.0020251 & \pm 0.0000005 & 9.91 & \pm 0.50 & -9 & 5 & 3443 & 580 \\
\hline $91500-34$ & 137 & 0.0020247 & \pm 0.0000005 & 9.73 & \pm 0.50 & -10 & 5 & 3429 & 540 \\
\hline $91500-35$ & 138 & 0.0020250 & \pm 0.0000005 & 9.88 & \pm 0.48 & -10 & 3 & 3469 & 543 \\
\hline $91500-36$ & 140 & 0.0020250 & \pm 0.0000005 & 9.87 & \pm 0.55 & -11 & 4 & 3488 & 583 \\
\hline $91500-37$ & 141 & 0.0020254 & \pm 0.0000005 & 10.08 & \pm 0.50 & -11 & 4 & 3491 & 623 \\
\hline $91500-38$ & 143 & 0.0020254 & \pm 0.0000005 & 10.07 & \pm 0.54 & -11 & 4 & 3462 & 434 \\
\hline 91500-39 & 144 & 0.0020245 & \pm 0.0000005 & 9.63 & \pm 0.54 & -11 & 4 & 3459 & 394 \\
\hline $91500-40$ & 146 & 0.0020251 & \pm 0.0000006 & 9.93 & \pm 0.58 & -10 & 3 & 3420 & 394 \\
\hline $91500-41$ & 147 & 0.0020245 & \pm 0.0000006 & 9.65 & \pm 0.56 & -10 & 4 & 3456 & 353 \\
\hline & & Weighted mean & $2 \sigma$ & Weighted mean & $2 \sigma$ & & & & \\
\hline 91500 & $n=39$ & 0.0020250 & \pm 0.0000002 & 9.86 & \pm 0.16 & & & & \\
\hline 91500 ref & & 0.0020250 & \pm 0.0000002 & 9.86 & \pm 0.22 & & & & \\
\hline OGC & & & & & & & & & \\
\hline$\overline{\mathrm{OGC}-01}$ & 6 & 0.0020180 & \pm 0.0000005 & 6.37 & \pm 0.50 & -14 & 5 & 1042 & 914 \\
\hline OGC-02 & 14 & 0.0020175 & \pm 0.0000005 & 6.15 & \pm 0.50 & -15 & 5 & 1006 & 891 \\
\hline OGC-03 & 22 & 0.0020169 & \pm 0.0000005 & 5.84 & \pm 0.46 & -16 & 5 & 933 & 879 \\
\hline OGC-04 & 31 & 0.0020174 & \pm 0.0000005 & 6.06 & \pm 0.47 & -16 & 5 & 893 & 867 \\
\hline OGC-05 & 40 & 0.0020170 & \pm 0.0000005 & 5.88 & \pm 0.48 & -15 & 6 & 564 & 920 \\
\hline OGC-06 & 49 & 0.0020169 & \pm 0.0000005 & 5.82 & \pm 0.46 & -15 & 5 & 530 & 916 \\
\hline OGC-07 & 58 & 0.0020171 & \pm 0.0000004 & 5.94 & \pm 0.43 & -16 & 5 & 575 & 766 \\
\hline OGC-08 & 76 & 0.0020170 & \pm 0.0000005 & 5.89 & \pm 0.49 & -16 & 5 & 538 & 804 \\
\hline OGC-09 & 94 & 0.0020158 & \pm 0.0000005 & 5.27 & \pm 0.53 & -18 & 5 & 918 & 550 \\
\hline OGC-10 & 112 & 0.0020167 & \pm 0.0000004 & 5.73 & \pm 0.43 & -13 & 6 & 1045 & 480 \\
\hline OGC-11 & 139 & 0.0020171 & \pm 0.0000005 & 5.92 & \pm 0.48 & -13 & 5 & 1181 & 608 \\
\hline OGC-12 & 145 & 0.0020173 & \pm 0.0000005 & 6.06 & \pm 0.48 & -11 & 6 & 1119 & 576 \\
\hline & & Weighted mean & & Weighted mean & $2 \sigma$ & & & & \\
\hline OGC & $n=12$ & 0.0020170 & \pm 0.0000003 & 5.91 & \pm 0.30 & & & & \\
\hline OGC ref. & & 0.0020170 & \pm 0.0000001 & 5.88 & \pm 0.06 & & & & \\
\hline Penglai & & & & & & & & & \\
\hline $\begin{array}{l}\text { Penglai-01 } \\
\end{array}$ & 15 & 0.0020149 & \pm 0.0000004 & 4.85 & \pm 0.43 & -13 & 6 & 2204 & 607 \\
\hline Penglai-02 & 32 & 0.0020155 & \pm 0.0000005 & 5.12 & \pm 0.48 & -13 & 5 & 2167 & 618 \\
\hline Penglai-03 & 50 & 0.0020150 & \pm 0.0000005 & 4.90 & \pm 0.52 & -13 & 5 & 2118 & 622 \\
\hline Penglai-04 & 67 & 0.0020153 & \pm 0.0000005 & 5.05 & \pm 0.48 & -13 & 5 & 2078 & 626 \\
\hline Penglai-05 & 85 & 0.0020160 & \pm 0.0000005 & 5.40 & \pm 0.48 & -14 & 5 & 2046 & 602 \\
\hline Penglai-06 & 103 & 0.0020153 & \pm 0.0000006 & 5.06 & \pm 0.57 & -13 & 4 & 2243 & 372 \\
\hline Penglai-07 & 142 & 0.0020150 & \pm 0.0000005 & 4.88 & \pm 0.53 & -13 & 4 & 2114 & 372 \\
\hline Penglai-08 & 130 & 0.0020150 & \pm 0.0000005 & 4.89 & \pm 0.51 & -13 & 4 & 2195 & 383 \\
\hline & & Weighted mean & $2 \sigma$ & Weighted mean & $2 \sigma$ & & & & \\
\hline Penglai & & 0.0020152 & \pm 0.0000004 & 5.02 & \pm 0.41 & & & & \\
\hline Penglai ref. & & 0.0020158 & \pm 0.0000001 & 5.31 & \pm 0.10 & & & & \\
\hline
\end{tabular}




\section{Session II}

\begin{tabular}{|c|c|c|c|c|c|c|c|c|c|}
\hline $\begin{array}{l}\text { Penglai-01 } \\
\end{array}$ & 1 & 0.0020159 & \pm 0.0000003 & 5.34 & \pm 0.29 & 21 & 22 & 2003 & 3456 \\
\hline Penglai-02 & 2 & 0.0020156 & \pm 0.0000003 & 5.21 & \pm 0.31 & 21 & 21 & 2109 & 3515 \\
\hline Penglai-03 & 3 & 0.0020156 & \pm 0.0000003 & 5.19 & \pm 0.29 & 19 & 21 & 2076 & 3436 \\
\hline Penglai-04 & 4 & 0.0020162 & \pm 0.0000003 & 5.49 & \pm 0.30 & 20 & 20 & 2146 & 3432 \\
\hline Penglai-05 & 5 & 0.0020160 & \pm 0.0000003 & 5.38 & \pm 0.30 & 19 & 20 & 2146 & 3392 \\
\hline Penglai-06 & 12 & 0.0020160 & \pm 0.0000003 & 5.40 & \pm 0.28 & 17 & 19 & 2146 & 3352 \\
\hline Penglai-07 & 13 & 0.0020159 & \pm 0.0000003 & 5.32 & \pm 0.32 & 17 & 19 & 2186 & 3352 \\
\hline Penglai-08 & 21 & 0.0020156 & \pm 0.0000003 & 5.21 & \pm 0.29 & 16 & 19 & 2146 & 3312 \\
\hline Penglai-09 & 22 & 0.0020155 & \pm 0.0000003 & 5.14 & \pm 0.30 & 16 & 19 & 2186 & 3312 \\
\hline Penglai-10 & 29 & 0.0020156 & \pm 0.0000003 & 5.19 & \pm 0.32 & 16 & 19 & 2166 & 3272 \\
\hline Penglai-11 & 30 & 0.0020163 & \pm 0.0000003 & 5.52 & \pm 0.32 & 17 & 17 & 2631 & 3424 \\
\hline Penglai-12 & 38 & 0.0020158 & \pm 0.0000003 & 5.28 & \pm 0.32 & 17 & 17 & 2671 & 3433 \\
\hline Penglai-13 & 39 & 0.0020157 & \pm 0.0000003 & 5.24 & \pm 0.29 & 16 & 18 & 2990 & 3021 \\
\hline Penglai-14 & 43 & 0.0020163 & \pm 0.0000003 & 5.51 & \pm 0.33 & 16 & 18 & 2946 & 2975 \\
\hline Penglai-15 & 44 & 0.0020157 & \pm 0.0000003 & 5.23 & \pm 0.32 & 16 & 18 & 2986 & 2975 \\
\hline Penglai & & $\begin{array}{r}0.0020158 \\
0.02 \text { mean }\end{array}$ & $\begin{array}{c}2 \sigma \\
\pm 0.0000002\end{array}$ & $\begin{array}{c}\text { Veighted mean } \\
5.31\end{array}$ & $\begin{array}{c}2 \sigma \\
\pm 0.17\end{array}$ & & & & \\
\hline Penglai ref. & & 0.0020158 & \pm 0.0000001 & 5.31 & \pm 0.10 & & & & \\
\hline
\end{tabular}

\begin{tabular}{|c|c|c|c|c|c|c|c|c|c|c|}
\hline M257-01 & & 7 & 0.0020343 & \pm 0.0000003 & 14.51 & \pm 0.29 & 9 & 18 & -2194 & 2002 \\
\hline M257-02 & & 15 & 0.0020339 & \pm 0.0000003 & 14.34 & \pm 0.33 & 8 & 17 & -2220 & 1964 \\
\hline M257-03 & & 31 & 0.0020341 & \pm 0.0000003 & 14.40 & \pm 0.33 & 7 & 17 & -2234 & 1779 \\
\hline M257-04 & & 41 & 0.0020335 & \pm 0.0000003 & 14.10 & \pm 0.31 & 7 & 18 & -2205 & 1728 \\
\hline M257-05 & & 42 & 0.0020336 & \pm 0.0000003 & 14.15 & \pm 0.29 & 8 & 18 & -2173 & 1694 \\
\hline M257 & $n=5$ & & $\begin{array}{r}\text { Weighted mean } \\
0.0020339\end{array}$ & $\begin{array}{c}2 \sigma \\
\pm 0.0000004\end{array}$ & $\begin{array}{c}\text { Weighted mean } \\
14.30\end{array}$ & $\begin{array}{c}2 \sigma \\
\pm 0.38\end{array}$ & & & & \\
\hline M257 ref. & & & 0.0020331 & \pm 0.0000002 & 13.93 & \pm 0.22 & & & & \\
\hline \multicolumn{11}{|l|}{ OGC } \\
\hline OGC-@01 & & 6 & 0.0020173 & \pm 0.0000003 & 6.04 & \pm 0.31 & 9 & 20 & -307 & -1122 \\
\hline OGC-@02 & & 14 & 0.0020176 & \pm 0.0000003 & 6.20 & \pm 0.33 & 8 & 19 & -348 & -1117 \\
\hline OGC-@03 & & 23 & 0.0020180 & \pm 0.0000003 & 6.38 & \pm 0.29 & 7 & 19 & -381 & -1114 \\
\hline OGC-@04 & & 32 & 0.0020177 & \pm 0.0000003 & 6.24 & \pm 0.31 & 7 & 20 & -290 & -1261 \\
\hline OGC-@05 & & 40 & 0.0020170 & \pm 0.0000003 & 5.87 & \pm 0.32 & 5 & 20 & -285 & -1304 \\
\hline \multirow[t]{2}{*}{ OGC-@06 } & & 45 & 0.0020176 & \pm 0.0000003 & 6.21 & \pm 0.29 & 7 & 20 & -609 & -1081 \\
\hline & & & Weighted mean & $2 \sigma$ & Weighted mean & $2 \sigma$ & & & & \\
\hline OGC & $n=6$ & & 0.0020175 & \pm 0.0000003 & 6.17 & \pm 0.32 & & & & \\
\hline OGC ref. & & & 0.0020170 & \pm 0.0000001 & 5.88 & \pm 0.06 & & & & \\
\hline
\end{tabular}

Note: Each $\delta^{18} \mathrm{O}$ error $(2 \sigma)$ represents the counting statistics errors for each individual spot and the external error based on all standards during the session, which were added in quadrature.

Field aperture centering digits are given and show little difference between standard and sample. mount. The location of the unknowns is Mount position $X Y$ for standard and sample indicates that most analyses are within the central region to standards.

Weighted average uncertainties represent 2 standard deviations of the mean. The corresponding solution data are from:

91500: Wiedenbeck et al. (2004); OGC: Petersson et al. (2019); Penglai: Li et al., 2011; M257: Nasdala et al., 2008.

* Sequential order of analyses.

${ }^{t}$ Raw ratios drift corrected using the standard analyses versus time. The instrumental mass fractionation, corrected using alpha SIMS

(ratio between the session average standard value and reference value).

${ }^{\S}$ Session I was normalised to a $\delta^{18} \mathrm{O}$ value of $9.86 \%$ for the 91500 standard and Session II to a $\delta^{18} \mathrm{O}$ value of $5.31 \%$ for the Penglai standi 
Table A5. Zircon Lu-Hf isotopes

\begin{tabular}{|c|c|c|c|c|c|c|c|c|c|c|c|c|c|c|c|c|c|c|c|c|}
\hline Spot I.D & $\begin{array}{l}\text { Corresponding } \\
\text { U-Pb spot I.D }\end{array}$ & Spot size & $\begin{array}{l}\text { No. } \\
\text { ratios } \\
\text { used }\end{array}$ & Comment & ${ }^{176} \mathrm{Yb} \mathbf{b}^{177} \mathrm{Hf}$ & $2 \sigma$ & ${ }^{176} \mathrm{Lu} /{ }^{177} \mathrm{Hf}$ & $2 \sigma$ & ${ }^{176} \mathrm{Hf} f^{177} \mathrm{Hf}$ & $2 \sigma$ & ${ }^{178} \mathrm{Hf} \mathrm{f}^{177} \mathrm{Hf}$ & $2 \sigma$ & $\begin{array}{l}\text { Interpreted } \\
\quad \text { age }\end{array}$ & $\sigma$ & $\begin{array}{c}{ }^{207} \mathrm{~Pb} / 206 \mathrm{~Pb} \\
\text { date }\end{array}$ & $\sigma$ & $\varepsilon_{\mathrm{Hft}}$ & $2 \sigma$ & $\delta^{18} \mathrm{O}$ & $2 \sigma$ \\
\hline Session 1 & & & & & & \pm & & \pm & & $\underline{I}$ & & \pm & (Ma) & \pm (Ma) & (Ma) & \pm (Ma) & & \pm & $(\%)$ & \pm (abs.) \\
\hline PR1_15TKPB17_01b & n5932_15TKPB17-01b & $040 \mu \mathrm{m}$ & $60 / 60$ & On U-Pb spo & 0.0127 & \pm 0.0003 & 0.000446 & \pm 0.000003 & 0.280343 & \pm .000018 & 1.467203 & \pm 0.000036 & 3704 & \pm 3 & 3704 & \pm 3 & -2.4 & \pm 0.6 & 6.04 & \pm 0.30 \\
\hline PR1_15TKPB17_02a & n5932_15TKPB17-02a & $40 \mu \mathrm{m}$ & $60 / 60$ & On U-Pb spo & 0.0289 & \pm 0.0017 & 0.001066 & \pm 0.000074 & 0.280388 & \pm .000025 & 1.467246 & \pm 0.000033 & 3632 & \pm 8 & 3632 & \pm 8 & -4.1 & \pm 0.9 & 6.04 & \pm 0.31 \\
\hline PR1_15TKPB17_03 & n5932_15TKPB17-03 & $40 \mu \mathrm{m}$ & $60 / 60$ & On U-Pb spo & 0.0371 & \pm 0.0017 & 0.001184 & \pm 0.000028 & 0.280372 & \pm .000029 & 1.467201 & \pm 0.000045 & 3732 & \pm 3 & 3732 & \pm 3 & -2.6 & \pm 1.0 & 5.24 & \pm 0.29 \\
\hline PR1_15TKPB17_04b & n5932_15TKPB17-04b & $040 \mu \mathrm{m}$ & $50 / 60$ & On U-Pb spo & 0.0199 & \pm 0.0006 & 0.000703 & \pm 0.000006 & 0.280379 & \pm .000023 & 1.467253 & \pm 0.000038 & 3710 & \pm 2 & 3710 & \pm 2 & -1.7 & \pm 0.8 & & \\
\hline PR1_15TKPB17_05 & n5932_15TKPB17-05 & $40 \mu \mathrm{m}$ & $60 / 60$ & On U-Pb spo & 0.0379 & \pm 0.0034 & 0.001270 & \pm 0.000113 & 0.280398 & \pm .000028 & 1.467242 & \pm 0.000040 & 3699 & \pm 1 & 3699 & \pm 1 & -2.7 & \pm 1.0 & & \\
\hline PR1_15TKPB17_14 & n5932_15TKPB 17-14 & $40 \mu \mathrm{m}$ & $60 / 60$ & On U-Pb spo & 0.0342 & \pm 0.0010 & 0.001136 & \pm 0.000046 & 0.280367 & \pm .000026 & 1.467242 & \pm 0.000042 & 3713 & \pm 5 & 3713 & \pm 5 & -3.1 & \pm 0.9 & & \\
\hline PR1_15TKPB17_15a & n5932_15TKPB17-15a & $40 \mu \mathrm{m}$ & $60 / 60$ & On U-Pb spo & 0.0296 & \pm 0.0007 & 0.001099 & \pm 0.000021 & 0.280375 & \pm .000028 & 1.467261 & \pm 0.000035 & 3729 & \pm 2 & 3729 & \pm 2 & -2.4 & \pm 1.0 & 5.11 & \pm 0.30 \\
\hline PR1_15TKPB17_15b & n5932_15TKPB17-15b & $040 \mu \mathrm{m}$ & $60 / 60$ & On U-Pb spo & 0.0197 & \pm 0.0004 & 0.000738 & \pm 0.000005 & 0.280356 & \pm .000019 & 1.467203 & \pm 0.000033 & 37 & \pm 2 & 373 & +2 & 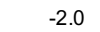 & \pm 0.7 & 5.26 & \pm 0.31 \\
\hline 7_17 & n5932_1 & $40 \mu \mathrm{m}$ & $60 / 60$ & On U-Pb spo & 0.0 & 5 & 0.000 & \pm 0.000040 & 0.28 & \pm .000024 & & & & \pm 3 & & & & & & 0.30 \\
\hline R1_15TKPB17_19 & n5932_1 & $40 \mu \mathrm{m}$ & $45 / 60$ & On U-Pb spo & & . 0031 & 0.000861 & \pm 0.000105 & 0.280639 & \pm .000023 & 1.467258 & 042 & & \pm 3 & & & & \pm 0.9 & & \pm 0.34 \\
\hline 1_15TKPB17_19b & n5932_15TKPB17-19 & $40 \mu \mathrm{m}$ & $60 / 60$ & Next to_19 & 0.0311 & \pm 0.0031 & 0.000998 & \pm 0.000109 & 0.280629 & \pm .000029 & 1.467248 & \pm 0.000031 & & \pm 3 & & \pm 3 & & \pm 1.1 & 5.56 & \pm 0.34 \\
\hline PR1_15TKPB17_41 & n5932_15TKPB17-41 & $40 \mu \mathrm{m}$ & $60 / 60$ & On U-P̄ spo & 0.0201 & \pm 0.0002 & 0.000689 & \pm 0.000012 & 0.280337 & \pm .000022 & 1.467210 & \pm 0.000042 & 3746 & \pm 3 & 3746 & \pm 3 & -2.3 & \pm 0.8 & 4.26 & \pm 0.30 \\
\hline PR1_15TKPB17_s10 & PR-10 ${ }^{-}$ & $40 \mu \mathrm{m}$ & $60 / 60$ & On U-Pb spo & 0.0268 & \pm 0.0014 & 0.000914 & \pm 0.000050 & 0.280342 & \pm .000024 & 1.467269 & \pm 0.000035 & 3696 & \pm 4 & 3696 & \pm 4 & -3.9 & \pm 0.9 & & \\
\hline PR3_15TKPB17_02 & 15tkpb17-02 & $40 \mu \mathrm{m}$ & $60 / 60$ & On U-Pb spo & 0.0156 & \pm 0.0006 & 0.000560 & \pm 0.000006 & 0.280323 & \pm .000021 & 1.467218 & \pm 0.000032 & 373 & \pm 2 & 373 & \pm 2 & -2.8 & \pm 0.8 & 5.59 & \pm 0.46 \\
\hline PR3_15TKPB17_13 & 15tkpb17-13 & $35 \mu \mathrm{m}$ & $60 / 60$ & On U-Pb spo & 0.0188 & \pm 0.0010 & 0.000631 & \pm 0.000019 & 0.280345 & \pm .000034 & 1.467216 & \pm 0.000055 & 368 & \pm 5 & 368 & \pm 5 & -3.4 & \pm 1.2 & & \\
\hline PR3_15TKPB17_14 & 15tkpb17-14 & $40 \mu \mathrm{m}$ & $60 / 60$ & On U-Pb spo & 0.0302 & \pm 0.0021 & 0.001039 & \pm 0.000089 & 0.280340 & \pm .000028 & 1.467247 & \pm 0.000041 & 3689 & \pm 5 & 3689 & \pm 5 & -4.4 & \pm 1.0 & & \\
\hline PR3_18APPB12_02 & 18appb12-02 & $40 \mu \mathrm{m}$ & $35 / 60$ & On U-Pb spo & 0.0439 & \pm 0.0035 & 0.001470 & \pm 0.000118 & 0.280406 & \pm .000027 & 1.467229 & \pm 0.000036 & 3753 & \pm 3 & 3753 & \pm 3 & -1.7 & \pm 1.0 & 4.57 & \pm 0.57 \\
\hline PR3_18APPB12_06 & 18appb12-06 & $40 \mu \mathrm{m}$ & $60 / 60$ & On U-Pb spo & 0.0228 & \pm 0.0018 & 0.000742 & \pm 0.000045 & 0.280424 & \pm .000024 & 1.467243 & \pm 0.000039 & 3463 & \pm 3 & 3463 & \pm 4 & -5.9 & \pm 0.9 & 6.57 & \pm 0.47 \\
\hline PR3_18APPB12_08 & 18appb12-08 & $40 \mu \mathrm{m}$ & $60 / 60$ & On U-Pb spo & 0.0044 & \pm 0.0001 & 0.000145 & \pm 0.000005 & 0.280578 & \pm .000025 & 1.467214 & \pm 0.000044 & 3451 & \pm 3 & 3442 & \pm 7 & 0.7 & \pm 0.9 & 6.10 & \pm 0.43 \\
\hline PR3_18APPB12_10 & 18appl & $40 \mu \mathrm{m}$ & $35 / 60$ & On U-Pb spo & 0.012 & \pm 0.0004 & 0.000419 & \pm 0.000016 & 0.280592 & \pm .000029 & 1.467299 & \pm 0.000059 & 34 & \pm 3 & 34 & \pm 4 & 0.6 & \pm 1.0 & 6.08 & \pm 0.55 \\
\hline 3-18TK & & 4 & $60 / 60$ & On & $0 . c$ & & 0 & 47 & 0.2 & & 1.4 & & & \pm 4 & & & -1 & & & 5 \\
\hline-187 & & & $60 / 60$ & & & & & & & & & & & \pm 3 & & \pm 3 & 1 & & & 9 \\
\hline & & & & & & & & & & & & & & \pm 4 & & \pm 4 & -3 & & & \\
\hline 601 01 & & 40 & $60 / 60$ & On U-Pb spo & 0.0 & \pm 0.0 & 0.00 & \pm 0.000 & & \pm .000 & & \pm 0 & 36 & \pm 3 & 3696 & \pm 3 & -2.9 & \pm 1.0 & 5.6 & \\
\hline PR3 18TKPB16 03 & 18appb16-03 & $40 \mu \mathrm{m}$ & $60 / 60$ & On U-Pb spo & 0.02 & \pm 0.0011 & 0.000914 & \pm 0.000038 & 0.280613 & \pm .000027 & 1.467283 & \pm 0.000040 & 3451 & \pm 3 & 3456 & \pm 3 & 0.0 & \pm 1.0 & 6.37 & $\begin{array}{l} \pm 0.49 \\
\pm 0\end{array}$ \\
\hline 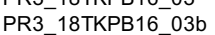 & 18appb16-03b & $40 \mu \mathrm{m}$ & $60 / 60$ & On U-Pb spo & 0.0490 & \pm 0.0016 & 0.001491 & \pm 0.000056 & 0.280409 & \pm .000027 & 1.467248 & \pm 0.000041 & 3451 & \pm 3 & 3446 & \pm 3 & 0.1 & \pm 1.0 & 6.11 & \pm 0.47 \\
\hline
\end{tabular}

Note. Present day value of $\mathrm{CHUR}{ }^{1 / 10} \mathrm{Hf} /{ }^{1 /} \mathrm{Hf}=0.282785$

$\lambda=1.867 \times 10^{-11}$

Case of

" $\varepsilon_{\text {Hft }}$ calculated using the interpreted age of the rock. 
Table A6. Zircon Lu-Hf standards

\begin{tabular}{|c|c|c|c|c|c|c|c|c|c|c|}
\hline & ${ }^{176} \mathrm{Hf} /{ }^{177} \mathrm{Hf}$ & $2 \sigma$ & ${ }^{178} \mathrm{Hf} /{ }^{177} \mathrm{Hf}$ & $2 \sigma$ & ${ }^{176} \mathrm{Lu} /{ }^{177} \mathrm{Hf}$ & $2 \sigma$ & ${ }^{176} \mathrm{Yb} /{ }^{177} \mathrm{Hf}$ & $2 \sigma$ & Total Hf (V) & Comment \\
\hline \multicolumn{11}{|l|}{ FC1 } \\
\hline & 0.282182 & \pm 0.000018 & 1.467212 & \pm 0.000028 & 0.001672 & \pm 0.000001 & 0.05259 & \pm 0.000276 & 21.4 & OK \\
\hline & 0.282167 & \pm 0.000016 & 1.467198 & \pm 0.000030 & 0.000630 & \pm 0.000000 & 0.01896 & \pm 0.000069 & 21.3 & OK \\
\hline & 0.282175 & \pm 0.000017 & 1.467216 & \pm 0.000025 & 0.001452 & \pm 0.000005 & 0.04499 & \pm 0.000465 & 23.4 & OK \\
\hline & 0.282185 & \pm 0.000018 & 1.467210 & \pm 0.000025 & 0.001458 & \pm 0.000019 & 0.04588 & \pm 0.000464 & 23.7 & OK \\
\hline & 0.282177 & \pm 0.000020 & 1.467229 & \pm 0.000018 & 0.001869 & \pm 0.000030 & 0.05898 & \pm 0.000754 & 23.1 & OK \\
\hline & 0.282201 & \pm 0.000022 & 1.467233 & \pm 0.000026 & 0.002681 & \pm 0.000014 & 0.08446 & \pm 0.000204 & 18.4 & OK \\
\hline & 0.282182 & \pm 0.000022 & 1.467223 & \pm 0.000022 & 0.001700 & \pm 0.000007 & 0.05331 & \pm 0.000285 & 17.9 & OK \\
\hline & 0.282185 & \pm 0.000018 & 1.467236 & \pm 0.000030 & 0.001215 & \pm 0.000004 & 0.03753 & \pm 0.000360 & 18.5 & OK \\
\hline & 0.282170 & \pm 0.000019 & 1.467235 & \pm 0.000030 & 0.000982 & \pm 0.000001 & 0.02961 & \pm 0.000160 & 19.4 & OK \\
\hline & 0.282182 & \pm 0.000019 & 1.467222 & \pm 0.000025 & 0.001184 & \pm 0.000004 & 0.03708 & \pm 0.000182 & 17.1 & OK \\
\hline Mean: & 0.282181 & \pm 0.000019 & 1.467221 & \pm 0.000026 & 0.001484 & \pm 0.000009 & 0.046339 & \pm 0.000322 & & \\
\hline Solution: & 0.282184 & $\begin{array}{l} \pm 0.000016 \\
\end{array}$ & & & & & & & & \\
\hline \multicolumn{11}{|l|}{91500} \\
\hline & 0.282316 & \pm 0.000022 & 1.467200 & \pm 0.000046 & 0.000453 & \pm 0.000000 & 0.01322 & \pm 0.000077 & 9.4 & OK \\
\hline & 0.282306 & \pm 0.000027 & 1.467250 & \pm 0.000036 & 0.000450 & \pm 0.000002 & 0.01333 & \pm 0.000105 & 9.2 & OK \\
\hline & 0.282312 & \pm 0.000020 & 1.467210 & \pm 0.000040 & 0.000327 & \pm 0.000001 & 0.00927 & \pm 0.000029 & 10.2 & OK \\
\hline & 0.282300 & \pm 0.000020 & 1.467222 & \pm 0.000040 & 0.000323 & \pm 0.000001 & 0.00967 & \pm 0.000201 & 9.7 & OK \\
\hline & 0.282311 & \pm 0.000024 & 1.467251 & \pm 0.000036 & 0.000321 & \pm 0.000002 & 0.00913 & \pm 0.000049 & 9.4 & OK \\
\hline & 0.282308 & \pm 0.000025 & 1.467250 & \pm 0.000039 & 0.000328 & \pm 0.000001 & 0.00941 & \pm 0.000083 & 9.4 & OK \\
\hline & 0.282326 & \pm 0.000026 & 1.467214 & \pm 0.000044 & 0.000332 & \pm 0.000001 & 0.00944 & \pm 0.000060 & 9.1 & OK \\
\hline Mean: & 0.282311 & \pm 0.000024 & 1.467228 & \pm 0.000040 & 0.000362 & \pm 0.000001 & 0.010495 & \pm 0.000086 & & \\
\hline Solution: & 0.282306 & 0.000008 & & & & & & & & \\
\hline
\end{tabular}




\begin{tabular}{|c|c|c|c|c|c|c|}
\hline & $0.280598 \pm 0.000019$ & $1.467227 \pm 0.000029$ & $0.000639 \pm 0.000035$ & $0.01767 \pm 0.001092$ & 16.9 & OK \\
\hline & $0.280655 \pm 0.000022$ & $1.467221 \pm 0.000030$ & $0.001649 \pm 0.000080$ & $0.04857 \pm 0.003229$ & 15.3 & OK \\
\hline & $0.280643 \pm 0.000022$ & $1.467222 \pm 0.000034$ & $0.001057 \pm 0.000008$ & $0.02880 \pm 0.000181$ & 16.7 & OK \\
\hline & $0.280590 \pm 0.000020$ & $1.467211 \pm 0.000026$ & $0.000674 \pm 0.000018$ & $0.01800 \pm 0.000623$ & 17.2 & OK \\
\hline & $0.280641 \pm 0.000018$ & $1.467259 \pm 0.000031$ & $0.001263 \pm 0.000049$ & $0.03705 \pm 0.002159$ & 15.2 & OK \\
\hline & $0.280626 \pm 0.000018$ & $1.467231 \pm 0.000030$ & $0.000728 \pm 0.000011$ & $0.01977 \pm 0.000272$ & 17.1 & OK \\
\hline & $0.280641 \pm 0.000023$ & $1.467229 \pm 0.000026$ & $0.001049 \pm 0.000012$ & $0.03082 \pm 0.000277$ & 14.4 & OK \\
\hline & $0.280629 \pm 0.000020$ & $1.467230 \pm 0.000034$ & $0.001058 \pm 0.000052$ & $0.02944 \pm 0.001469$ & 14.5 & OK \\
\hline & $0.280630 \pm 0.000022$ & $1.467220 \pm 0.000030$ & $0.001434 \pm 0.000066$ & $0.04201 \pm 0.002580$ & 14.5 & OK \\
\hline & $0.280691 \pm 0.000022$ & $1.467232 \pm 0.000039$ & $0.001903 \pm 0.000065$ & $0.05508 \pm 0.002567$ & 13.3 & OK \\
\hline Mean: & $0.280634 \pm 0.000021$ & $1.467228 \pm 0.000031$ & $0.001145 \pm 0.000040$ & $0.032721 \pm 0.001445$ & & \\
\hline Solution: & $0.280633 \pm 0.000034$ & & & & & \\
\hline \multicolumn{7}{|l|}{ Mud Tank } \\
\hline & $0.282482 \pm 0.000011$ & $1.467251 \pm 0.000027$ & $0.000011 \pm 0.000000$ & $0.00033 \pm 0.000006$ & 18.8 & OK \\
\hline & $0.282478 \pm 0.000014$ & $1.467220 \pm 0.000025$ & $0.000008 \pm 0.000000$ & $0.00026 \pm 0.000008$ & 18.3 & OK \\
\hline & $0.282490 \pm 0.000013$ & $1.467231 \pm 0.000027$ & $0.000008 \pm 0.000000$ & $0.00027 \pm 0.000007$ & 18.6 & OK \\
\hline & $0.282491 \pm 0.000013$ & $1.467257 \pm 0.000025$ & $0.000010 \pm 0.000000$ & $0.00033 \pm 0.000006$ & 18.8 & OK \\
\hline & $0.282480 \pm 0.000011$ & $1.467251 \pm 0.000027$ & $0.000008 \pm 0.000000$ & $0.00026 \pm 0.000007$ & 18.5 & OK \\
\hline & $0.282488 \pm 0.000014$ & $1.467228 \pm 0.000027$ & $0.000009 \pm 0.000000$ & $0.00027 \pm 0.000009$ & 18.0 & OK \\
\hline & $0.282494 \pm 0.000016$ & $1.467259 \pm 0.000026$ & $0.000008 \pm 0.000000$ & $0.00025 \pm 0.000009$ & 18.5 & OK \\
\hline & $0.282478 \pm 0.000013$ & $1.467222 \pm 0.000026$ & $0.000008 \pm 0.000000$ & $0.00026 \pm 0.000007$ & 17.6 & OK \\
\hline & $0.282488 \pm 0.000013$ & $1.467223 \pm 0.000031$ & $0.000008 \pm 0.000000$ & $0.00026 \pm 0.000006$ & 18.0 & OK \\
\hline & $0.282487 \pm 0.000014$ & $1.467232 \pm 0.000032$ & $0.000008 \pm 0.000000$ & $0.00026 \pm 0.000007$ & 18.2 & OK \\
\hline & $0.282487 \pm 0.000017$ & $1.467237 \pm 0.000023$ & $0.000010 \pm 0.000000$ & $0.00032 \pm 0.000007$ & 18.1 & OK \\
\hline & $0.282493 \pm 0.000014$ & $1.467239 \pm 0.000029$ & $0.000008 \pm 0.000000$ & $0.00027 \pm 0.000008$ & 18.3 & OK \\
\hline & $0.282493 \pm 0.000014$ & $1.467224 \pm 0.000029$ & $0.000008 \pm 0.000000$ & $0.00027 \pm 0.000008$ & 18.5 & OK \\
\hline & $0.282496 \pm 0.000014$ & $1.467219 \pm 0.000027$ & $0.000008 \pm 0.000000$ & $0.00026 \pm 0.000007$ & 18.7 & OK \\
\hline & $0.282489 \pm 0.000013$ & $1.467233 \pm 0.000029$ & $0.000008 \pm 0.000000$ & $0.00026 \pm 0.000007$ & 18.2 & OK \\
\hline Mean: & $0.282488 \pm 0.000014$ & $1.467235 \pm 0.000027$ & $0.000009 \pm 0.000000$ & $0.000276 \pm 0.000007$ & & \\
\hline
\end{tabular}

$0.280655 \pm 0.000022 \quad 1.467221 \pm 0.000030 \quad 0.001649 \pm 0.000080$

$0.001057+0.000008$

$280590 \pm 0.000020 \quad 1.467211 \pm 0.000026 \quad 0.000674 \pm 0.000018$

$0.04201+0.002580$

$0.05508 \pm 0.002567$

$0.280544 \pm 0.000022$

$0.280573 \pm 0.000022$

$0.280544 \pm 0.000020$

$0.280556 \pm 0.000019$ $0.280578 \pm 0.000018$ $0.280571 \pm 0.000023$ $0.280558 \pm 0.000021$ $0.280534 \pm 0.000022$ $\pm 0.000023$ $0.280558 \pm 0.000021$ $0.280554 \pm 0.000007$

\section{Solution: $0.282507 \pm 0.000006$}

Note: Summary of Lu-Hf-Yb isotope data measured from reference zircons during the course of this study by laser ablation MC-ICPMS, where Hf isotope ratios are session normalized to Mud Tank zircon ${ }^{176} \mathrm{Hf} /{ }^{177} \mathrm{Hf}=0.282507$ (Woodhead and Hergt, 2005, itself reported relative to $\mathrm{JMC} 475{ }^{176} \mathrm{Hf} /{ }^{177} \mathrm{Hf}=0.282160$ ). Uncertainties represent 2 standard deviations of the mean. The corresponding solution data are from:

FC-1: ${ }^{176} \mathrm{Hf} /{ }^{177} \mathrm{Hf}=0.282184 \pm 0.000016$, Temora $2{ }^{176} \mathrm{Hf} /{ }^{177} \mathrm{Hf}=0.282686 \pm 0.000008,91500{ }^{176} \mathrm{Hf} /{ }^{177} \mathrm{Hf}=0.282306 \pm 0.000008(\mathrm{Woodhead}$ and $\mathrm{Hergt}, 2005)$, and $O G C{ }^{176} \mathrm{Hf} /{ }^{177} \mathrm{Hf}=0.280633 \pm 0.000034$ (Kemp et al., 2017). All reported relative to JMC475 ${ }^{176} \mathrm{Hf} /{ }^{177} \mathrm{Hf}=0.282160$. 